\title{
Capsular glycan recognition provides antibody-mediated immunity against tuberculosis
}

\author{
Tingting Chen, ${ }^{1}$ Caroline Blanc, ${ }^{1}$ Yanyan Liu, ${ }^{1}$ Elise Ishida, ${ }^{1}$ Sarah Singer, ${ }^{1}$ Jiayong Xu, ${ }^{1}$ Maju Joe, ${ }^{2}$ Elizabeth R. Jenny-Avital, ${ }^{1}$ \\ John Chan, ${ }^{1,3}$ Todd L. Lowary, ${ }^{2}$ and Jacqueline M. Achkar ${ }^{1,3}$ \\ 'Department of Medicine, Albert Einstein College of Medicine, Bronx, New York, USA. ${ }^{2}$ Alberta Clycomics Centre and Department of Chemistry, University of Alberta, Edmonton, Alberta, Canada. \\ ${ }^{3}$ Department of Microbiology and Immunology, Albert Einstein College of Medicine, Bronx, New York, USA.
}

\begin{abstract}
A better understanding of all immune components involved in protecting against Mycobacterium tuberculosis infection is urgently needed to inform strategies for novel immunotherapy and tuberculosis (TB) vaccine development. Although cell-mediated immunity is critical, increasing evidence supports that antibodies also have a protective role against TB. Yet knowledge of protective antigens is limited. Analyzing sera from 97 US immigrants at various stages of $M$. tuberculosis infection, we showed protective in vitro and in vivo efficacy of polyclonal IgG against the $M$. tuberculosis capsular polysaccharide arabinomannan (AM). Using recently developed glycan arrays, we established that anti-AM IgC induced in natural infection is highly heterogeneous in its binding specificity and differs in both its reactivity to oligosaccharide motifs within AM and its functions in bacillus Calmette-Guérin vaccination and/or in controlled (latent) versus uncontrolled (TB) M. tuberculosis infection. We showed that anti-AM IgC from asymptomatic but not from diseased individuals was protective and provided data suggesting a potential role of IgC2 and specific AM oligosaccharides. Filling a gap in the current knowledge of protective antigens in humans, our data support the key role of the M. tuberculosis surface glycan AM and suggest the importance of targeting specific glycan epitopes within AM in antibody-mediated immunity against TB.
\end{abstract}

\section{Introduction}

Active tuberculosis (TB), the clinical manifestation of uncontrolled Mycobacterium tuberculosis (Mtb) infection, is now the leading cause of death from a single pathogen worldwide. A better understanding of all immune components involved in protection against TB is urgently needed to inform the development of novel immunotherapies and effective vaccines against TB (1). Currently, an estimated 10 million new TB cases occur annually, with 1.6 million associated deaths (2). As numbers of multiple-drug-resistant $\mathrm{TB}$ cases are rising, improving global control of this transmissible respiratory disease becomes even more critical (2). With around 1.7 billion people, about $25 \%$ of the world's population, infected with Mtb, the reservoir of individuals at risk for developing TB is enormous (3). Although the lifetime risk for disease development is around $5 \%-10 \%$, most ostensibly immunocompetent Mtb-infected individuals can control their infection in the form of asymptomatic latent Mtb infection (LTBI) $(2,3)$. However, the immune components involved in controlling or protecting against Mtb infection remain incompletely understood (reviewed in refs. 4,5 ).

Although cell-mediated immunity plays a pivotal role, the humoral immune response and other arms of the immune system also contribute to the protection against TB (reviewed

Conflict of interest: The authors have declared that no conflict of interest exists. Copyright: () 2020, American Society for Clinical Investigation.

Submitted: March 11, 2019; Accepted: January 3, 2020; Published: March 3, 2020. Reference information: / Clin Invest. 2020;130(4):1808-1822.

https://doi.org/10.1172/JCl128459. in refs. 4-8). The only currently available vaccine against TB, based on the attenuated Mycobacterium bovis bacillus Calmette-Guérin (BCG) strain, as well as newly developed vaccines, targets cell-mediated immunity. However, their efficacy in humans, despite recent improvements with novel vaccines and BCG revaccination, is not optimal $(4,9,10)$. Vaccines eliciting responses from both arms of adaptive immunity, cell-mediated and antibody-mediated (Ab-mediated), may work synergistically given their many interactions with the other immune arms. Evidence for this comes from our recent murine immunization studies showing that the induction of both is superior to either alone (11). Because of the long-standing conviction that Mtb, a predominantly intracellular pathogen, is outside the reach of extracellularly located Abs, Ab functions against Mtb have been insufficiently studied. However, Abs can protect against intracellular pathogens, including Mtb, through mechanisms such as interactions with innate immune cells (reviewed in refs. 5, 12-14). Recent studies provide compelling evidence for human Ab functions against Mtb (15-19). Both in vitro (17) and in vivo (19) studies show that serum Abs from asymptomatic Mtb-exposed and/or -infected individuals, but not from TB patients, are protective. Because of the use of either total IgG or polyclonal IgG against mycobacterial multiantigen preparations, conclusions about specific protective antigens could not be made. These data highlight the need for investigations of human Abs specific to a single antigen.

Abs against capsular and other surface polysaccharides (PSs; also referred to as glycans) of extra- and intracellular pathogens 


\section{Table 1. Demographics and clinical variables of study groups}

\begin{tabular}{|c|c|c|c|c|c|c|}
\hline \multicolumn{2}{|c|}{$\begin{array}{l}\text { Demographics and clinical } \\
\text { variables }\end{array}$} & $\begin{array}{c}\mathrm{TST}^{-} \\
(n=36)\end{array}$ & $\begin{array}{c}\mathrm{TST}^{+} \mathrm{IGRA}^{-} \\
(n=24)\end{array}$ & $\begin{array}{c}\mathrm{TST}^{+} \mathrm{IGRA}^{+} \\
(n=14)\end{array}$ & $\begin{array}{c}\text { TB } \\
(n=23)\end{array}$ & $P$ \\
\hline \multicolumn{2}{|c|}{ Male, no. (\%) } & $14(39)$ & $13(54)$ & $7(50)$ & $14(61)$ & $0.388^{A}$ \\
\hline \multicolumn{2}{|c|}{ Age, yr, mean (SD) } & 34 (9) & $37(11)$ & $39(12)$ & $48(17)$ & $0.001^{\mathrm{B}}$ \\
\hline \multicolumn{2}{|c|}{ Non-US born, ${ }^{\mathrm{C}}$ no. (\%) } & $16(44)$ & $21(88)$ & $13(93)$ & $19(83)$ & $0.0001^{A}$ \\
\hline \multirow[t]{5}{*}{ Race, no. } & White & 20 & 7 & 2 & 0 & $<0.001^{\circ}$ \\
\hline & Black & 0 & 4 & 0 & 7 & \\
\hline & Asian & 8 & 8 & 7 & 6 & \\
\hline & Hispanic & 7 & 5 & 5 & 8 & \\
\hline & Other & 1 & 0 & 0 & 2 & \\
\hline \multicolumn{2}{|c|}{ History of BCG vaccination, no. (\%) } & $16(44)$ & $21(88)$ & $13(93)$ & $19(83)$ & $<0.001^{\mathrm{A}}$ \\
\hline \multicolumn{2}{|c|}{ Smear ${ }^{\mathrm{E}}$ positive, no. (\%) } & NA & NA & NA & $9(39)$ & \\
\hline \multicolumn{2}{|c|}{ Disseminated TB, ${ }^{\mathrm{F}}$ no. (\%) } & NA & NA & NA & $11(48)$ & \\
\hline
\end{tabular}

${ }^{A} \chi^{2}$ test. ${ }^{B}$ One-way ANOVA. 'Subjects emigrated from various TB-endemic regions,

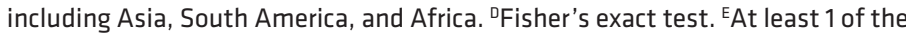
initial 3 sputum smears was positive for acid-fast bacilli. ${ }^{\text {F}}$ Disseminated TB was defined as evidence for extrapulmonary TB and/or miliary TB on radiographic imaging. TST ${ }^{-}$, tuberculin skin test-negative asymptomatic subjects; TST $^{+}$IGRA- or $\mathrm{TST}^{+}$IGRA ${ }^{+}$, tuberculin skin test-positive, IFN- $\gamma$ release assay-negative or -positive asymptomatic subjects; TB, patients with culture-confirmed active tuberculosis. tective efficacy in humans. Both BCG vaccination and Mtb infection in humans elicit serum Abs against AM and LAM $(15,16,30,35)$. We recently showed that vaccination of Mtb-uninfected young adults (living in the United Kingdom) with the same BCG strain (Danish) elicits not only greatly variable anti-AM IgG titers, but also IgG highly heterogeneous in its binding specificity to AM OS motifs within the PS (16). Although we and others have shown that serum IgG titers against AM and LAM are significantly associated with intracellular in vitro functions of innate immune cells against $\mathrm{Mtb}$ when coincubated with sera $(15,16)$, our prior studies also suggest a role of specific AM OS motifs (16). However, the data were based on associating functions against BCG with anti-AM IgG titer in BCG immune sera. To assess our hypothesized key role of AM and its epitopes in human Mtb infection, the main objectives of the current study were (a) to characterize the IgG responses to native Mtb capsular AM and synthetic AM OS motifs in a large cohort of HIV-negative individuals at various stages of Mtb infection; and (b) to determine the protective functions and efficacy of human anti-AM polyclonal IgG against Mtb in vitro and in vivo. are known to be protective and are the immune correlates of some of our most successful vaccines (reviewed in refs. 20-22). The capsule of Mtb is an important virulence factor and consists largely of proteins and PSs (23-26). The major Mtb capsular PSs are $\alpha$-glucan (70\%-80\%) and arabinomannan (AM; 10\%-20\%). Our work is focused on capsular AM, which, when lipidated, is also a component of the outer membrane/cell wall as part of immunomodulatory lipoarabinomannan (LAM) (23-26). The arabinan domains of both AM and LAM are composed solely of $\mathrm{D}$-arabinose residues in the 5-membered (furanose) ring form (Araf; ref. 26). Humans do not produce oligosaccharides (OSs) with furanose residues. Hence OSs containing Ara $f$ are highly immunogenic, and Abs that recognize AM and LAM typically do so through recognition of Araf residues (16, 27-29). By contrast, $\alpha$-glucan, the main mycobacterial capsular PS, is very similar in structure to glycogen and starch, both components of the human diet. This similarity might explain the low Ab responses to $\alpha$-glucan found in our prior studies with Mtb-infected humans (30). In contrast to murine mAbs recognizing $\alpha$-glucan, passive murine transfer studies with some (but not all) mAbs against AM/LAM have shown moderate protective efficacy (31, 32), and murine immunization studies with AM-containing glycoconjugates by us and others have shown moderate protection in Mtb-infected mice $(11,33,34)$. However, despite being a valid model, mice differ from humans in TB pathogenesis and their immune response to Mtb. Therefore, detailed explorations of the heterogeneous humoral immune response to Mtb in humans are warranted.

PSs are complex molecules that can include determinants eliciting protective, nonprotective, or even disease-enhancing Abs (reviewed in ref. 20). Thus, elucidating the diversity of humoral immune responses to Mtb surface glycans is critical to determine the key glycan epitopes that could render pro-

\section{Results}

IgG responses to $A M$ at various stages of human $M t b$ infection vary widely. Subject groups included mostly US immigrants from diverse TB-endemic regions who were HIV uninfected and either were asymptomatic or had symptomatic culture-confirmed active TB (Table 1). Asymptomatic subjects were categorized by tuberculin skin test (TST) results into $\mathrm{TST}^{-}(n=36)$ and $\mathrm{TST}^{+}(n=38)$. A second diagnostic test, the whole-blood IFN- $\gamma$ release assay (IGRA), was performed when the initial test (TST) was positive, and a subject was considered Mtb infected if both tests were positive (36). Nevertheless, a TST ${ }^{+} \mathrm{IGRA}^{-}$subject could have still been infected if the risk of remote Mtb exposure was high, such as in immigrants from TB-endemic regions (36). Therefore, rather than categorizing subjects into LTBI and non-LTBI, we classified TST $^{+}$ subjects as $\mathrm{TST}^{+} \mathrm{IGRA}^{-}(n=24)$ and $\mathrm{TST}^{+} \mathrm{IGRA}^{+}(n=14)$. Patients with TB $(n=23)$ had either localized pulmonary or disseminated culture-confirmed TB (Table 1).

Regardless of the stage of Mtb infection, serum IgG reactivity to native AM varied broadly in all groups (Figure 1). Patients with TB had significantly higher IgG reactivity to AM compared with asymptomatic subjects who were either $\mathrm{TST}^{-}$or $\mathrm{TST}^{+} \mathrm{IGRA}^{+}$(Figure 1A). For IgG subclass responses, focusing on comparing the 2 groups TB versus $\mathrm{TST}^{+} \mathrm{IGRA}^{+}$, this included significantly higher anti-AM IgG1 and IgG3 titers but only a trend $(P=0.08)$ for higher IgG2 titers in the TB group (Figure 1, B-D). Anti-AM IgG1 and IgG2 were the 2 major subclasses elevated in all groups, whereas IgG3 levels were lower and IgG4 was rarely detected (Supplemental Figure 1; supplemental material available online with this article; https://doi.org/10.1172/JCI128459DS1). Interestingly, the anti-AM IgG2/IgG1 subclass ratio was significantly higher in $\mathrm{TST}^{+}$ IGRA $^{+}$subjects (median 1.3, IQR 0.8-2.1) compared with TB (median 0.6, IQR 0.3-1.2; $P=0.01$ ). Among TB patients, those with localized pulmonary disease had a trend for higher anti-AM 
A

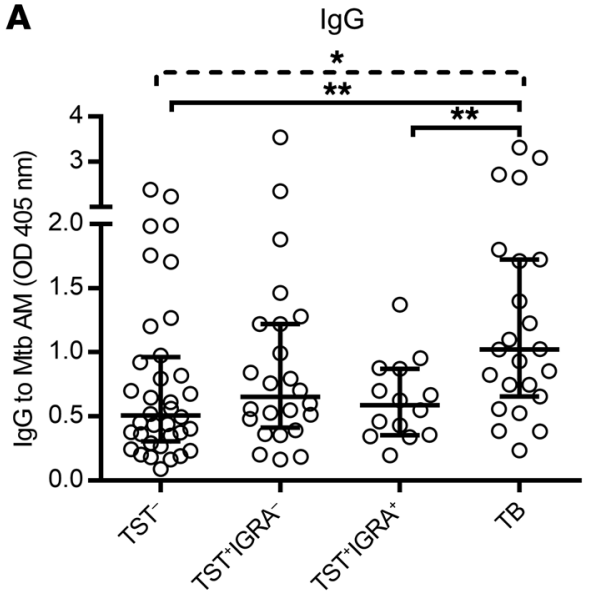

C

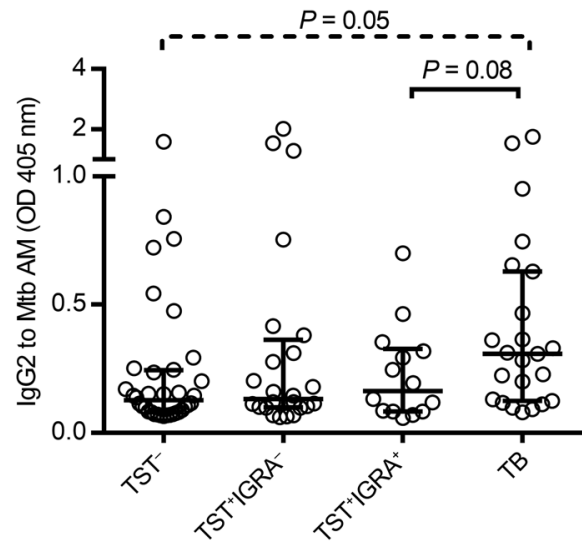

B

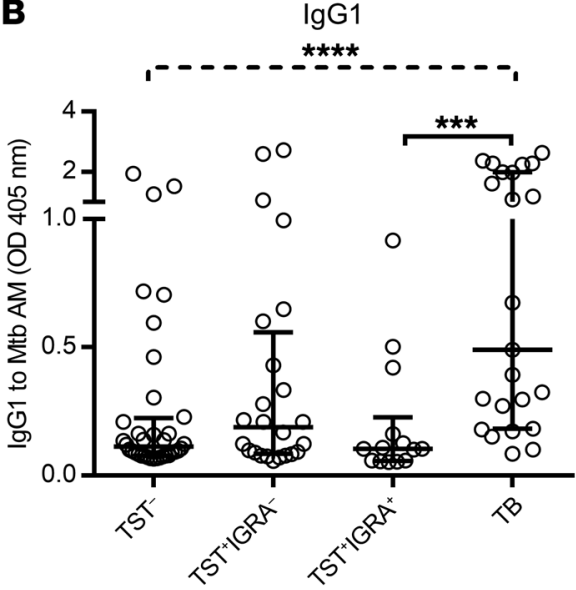

D

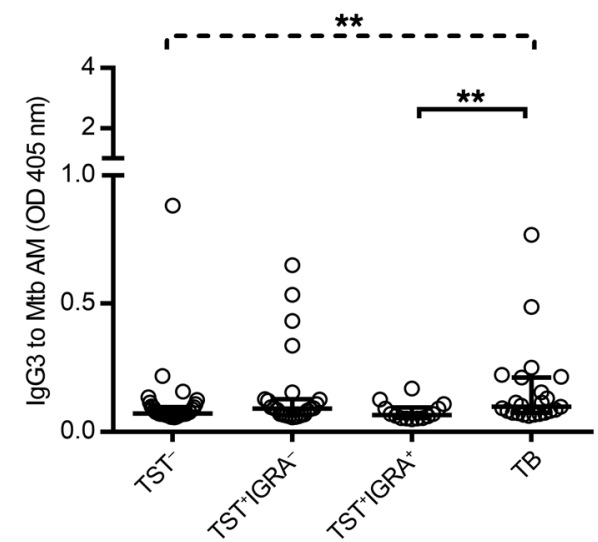

Figure 1. Wide range of IgG responses to Mtb (H37Rv) capsular AM at various stages of human Mtb infection. Comparison of anti-AM IgG (A) and IgG subclasses 1-3 (B-D) between sera (1:50) from asymptomatic subjects: TST $^{-}$ $(n=36), \mathrm{TST}^{+}\left|\mathrm{GRA}^{-}(n=24), \mathrm{TST}^{+}\right| \mathrm{GRA}^{+}(n=$ $14)$, and symptomatic TB patients $(n=23)$. Dashed lines represent Kruskal-Wallis test. Solid lines represent Mann-Whitney $U$ test. Two-group comparisons for IgC subclasses contrast TST ${ }^{+}$IGRA ${ }^{+}$versus TB. Lines and error bars represent medians with interquartile ranges (IQRs). ${ }^{*} P<0.05,{ }^{* *} P<0.01,{ }^{* * *} P<$ $0.001,{ }^{* * *} P<0.0001$. TB, active tuberculosis.
IgG titers compared with those with disseminated disease $(P=$ 0.07; Figure 2A). Although the IgG1 titers between these 2 forms of disease were not significantly different (Figure 2B), anti-AM IgG2 titers were significantly higher in localized pulmonary compared with disseminated TB (Figure 2C).

Human polyclonal IgG has a high level of cross-reactivity to capsular AM from strains of the Mtb complex group. To further assess whether human polyclonal IgG reactivity to AM cross-reacted between Mtb strains or BCG, we isolated capsular AM from 3 Mtb laboratory strains (H37Rv, the avirulent H37Ra, and Erdman), a clinical strain (CDC1551), and a BCG vaccine strain (Pasteur). We observed high and significant correlations between reactivity to capsular $\mathrm{AM}$ isolated from $\mathrm{H} 37 \mathrm{Rv}$ and reactivity to that isolated from the other strains, regardless of subject group with or without evidence of Mtb infection $(r=0.74-0.98, P<$ 0.01-0.0001; Supplemental Figure 2). These findings were consistent with equally strong correlations seen in our prior study comparing anti-AM IgG, derived from newly BCG-vaccinated adults, with capsular AM isolated from BCG (Pasteur) versus Mtb (H37Rv and H37Ra) (16).

Anti-AM IgG has opsonophagocytic activity in controlled but not in uncontrolled Mtb infection. As previously shown by us and others, the increase in serum anti-AM IgG titer after BCG vaccination correlated significantly with enhanced BCG phagocytosis in human macrophages coincubated with the corresponding sera $(15,16)$. To validate this association for Mtb with biologically independent samples from humans with or without Mtb infection, and then to show the key role of targeting AM in Ab-mediated immunity against TB, we first tested whether coincubation of heat-inactivated sera with moderate to high anti-AM IgG titers (OD > 0.4) enhanced Mtb phagocytosis by human macrophages. We focused on assessing macrophage functions in the presence and absence of antigen-specific IgG because these innate immune cells are the main host site for Mtb infection, persistence, and growth $(37,38)$. In both Mtb infection groups (TST ${ }^{+} \mathrm{IGRA}^{+}$and $\mathrm{TB}$ ), anti-AM IgG titers correlated significantly with Mtb phagocytosis by THP-1-derived human macrophages $\left(P=0.015\right.$ for $\mathrm{TST}^{+} \mathrm{IGRA}^{+}$and $P=0.001$ for TB; Figure 3, A and D). When analyzing subclass responses, we found that in the TB group both anti-AM IgG1 and IgG2 correlated significantly with Mtb phagocytosis; in the $\mathrm{TST}^{+} \mathrm{IGRA}^{+}$ group this correlation was only seen for IgG2 (Figure 3, B, C, $\mathrm{E}$, and $\mathrm{F}$ ). However, no association was observed with anti-AM IgG in the Mtb-uninfected groups $(r=0.09, P=0.77$, among 14 TST $^{-}$subjects; and $r=0.37, P=0.16$, among 16 TST $^{+}$IGRA $^{-}$ subjects). We therefore focused our functional evaluations on the $2 \mathrm{Mtb}$ infection groups $\mathrm{TST}^{+} \mathrm{IGRA}^{+}$and TB. However, a few BCG-vaccinated TST $^{-}$subjects $(2 / 36)$ and BCG-vaccinated TST $^{+}$IGRA $^{-}$subjects $(2 / 24)$ had exceptionally high anti-AM IgG titer (OD > 2; Figure 1). One of the 2 TST $^{-}$subjects had sufficient serum quantity available and was therefore included in our mycobacterial growth inhibition assay. Because both of the 
A

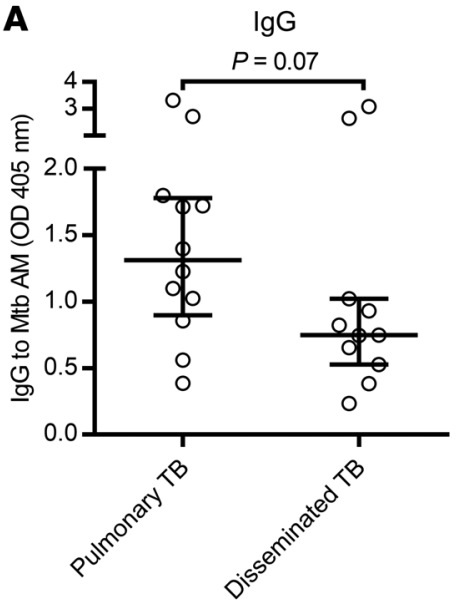

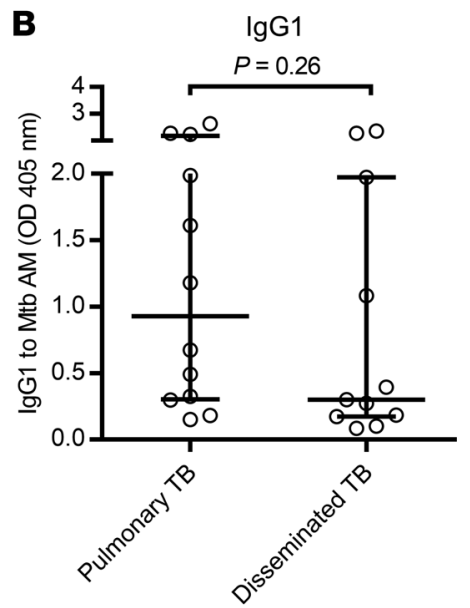

Figure 2. Patients with disseminated TB have significantly lower IgG2 reactivity to Mtb capsular AM. Comparison of IgG (A) and IgG subclass (B and C) responses to capsular AM (H37Rv) between TB patients with localized pulmonary $(n=12)$ and those with disseminated disease ( $n=11)$. Sera were tested at 1:50 dilutions. Mann-Whitney $U$ test. Lines and error bars represent medians with IQRs.

high-titer $\mathrm{TST}^{+} \mathrm{IGRA}^{-}$subjects were immigrants from India and had a history of potential Mtb exposure and sufficient serum available, they were included in all of our functional studies.

To show the causal role of anti-AM IgG in the enhancement of Mtb phagocytosis and other functions, we next depleted sera for anti-AM Abs and reevaluated their effects on human macrophages. Using initially the sera with the highest anti-AM IgG titers from 4 asymptomatic subjects $\left(2 \mathrm{TST}^{+} \mathrm{IGRA}^{+}\right.$and $2 \mathrm{TST}{ }^{+} \mathrm{IG}^{-}$ $\mathrm{RA}^{-}$), we first demonstrated that depletion of antigen-specific Abs via AM-coupled Dynabeads substantially reduced the IgG reactivity to Mtb capsular AM, measured by ELISA (Figure 4A).
We further show IgG binding of all 4 high-titer sera to the surface of Mtb (grown without detergent to preserve the capsule) with remarkable reduction of such binding after anti-AM Ab depletion, thereby demonstrating that Mtb surface-binding serum IgG reacted strongly with capsular AM of whole bacteria (Figure 4B). Consistent with this finding is the observation that Mtb phagocytosis by human macrophages (derived from THP-1 cells) was proportionally reduced when the cells were coincubated with anti-AM Ab-depleted compared with nondepleted sera (Figure 4C). These data demonstrate that the opsonophagocytic activity was due to Abs against AM.
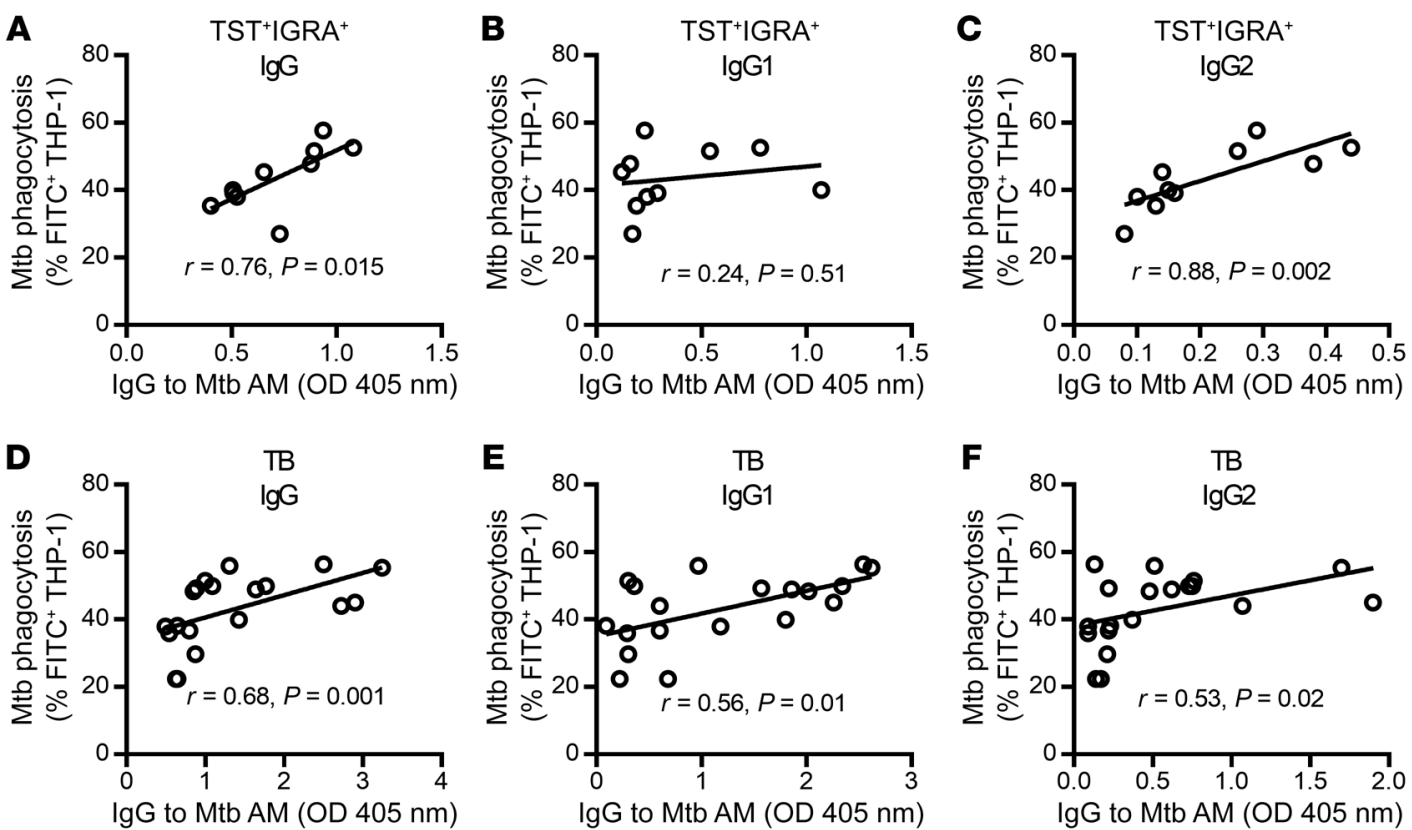

Figure 3. Significant correlation between Mtb phagocytosis and anti-AM IgG and subclass responses in subjects with Mtb infection. Correlations of antiAM IgC and subclass reactivity and Mtb (H37Ra; MOI 20) phagocytosis by THP-1-derived human macrophages incubated with heat-inactivated sera (10\%) from asymptomatic TST+|IRRA subjects, $n=10$ (A-C), and TB patients, $n=19$ (D-F). Tested sera (1:50) had moderate to high anti-AM IgG titers (OD >0.4 for TST+IGRA ${ }^{+}$subjects, and OD > 0.5 for TB patients). Representative data from 2 experiments. Spearman's rank correlation. 
A

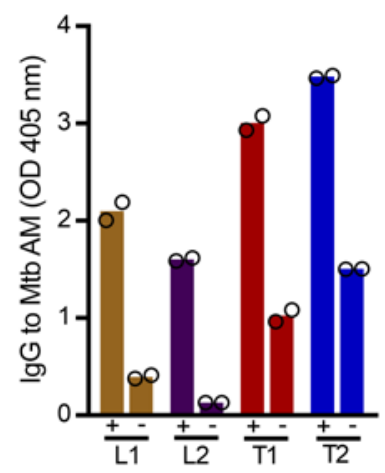

B

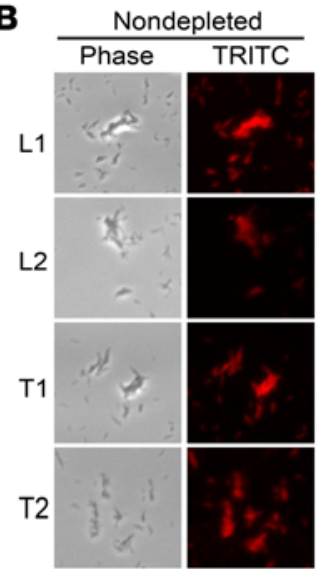

Depleted

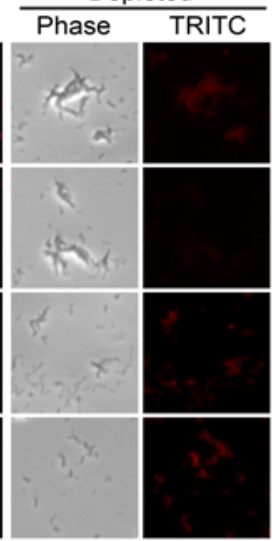

C

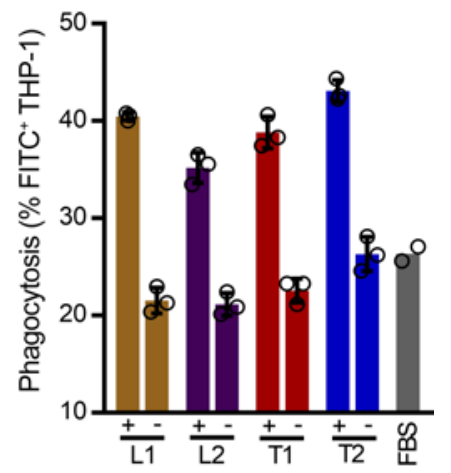

D

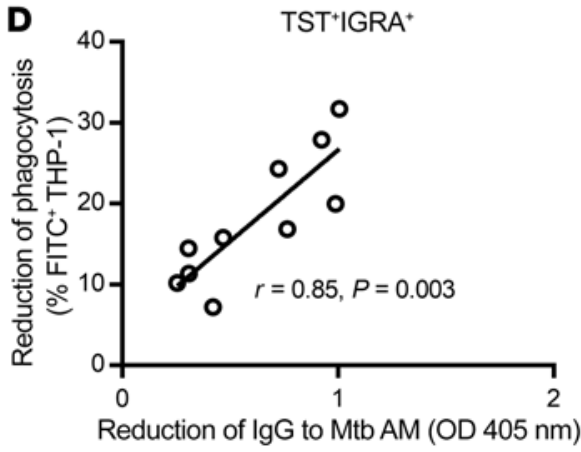

E

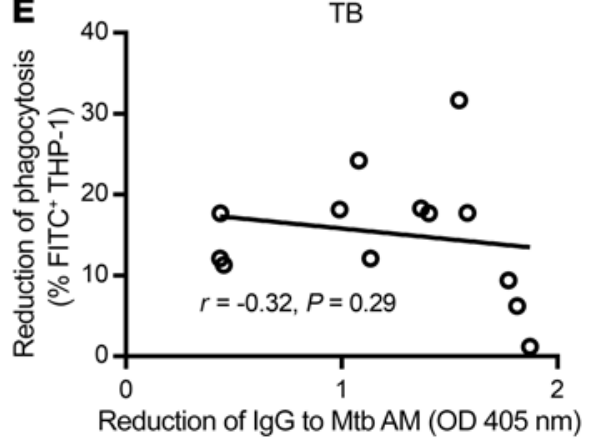

Figure 4. Mtb phagocytosis is anti-AM Ab mediated in TST+IGRA- and TST+IGRA+ subjects but not those with TB. (A) Effects of anti-AM IgG titers from $2 \mathrm{TST}^{+} \mathrm{IGRA}^{+}(\mathrm{L})$ and $2 \mathrm{TST}^{+} \mathrm{IGRA}^{-}(\mathrm{T})$ high-titer subjects before (+) and after (-) anti-AM Ab depletion. Columns represent mean of duplicates (circles). Notably, the depletion of the highest-titer serum (T2, a TST+IGRA- subject) remained incomplete despite several depletion rounds. Sera were tested at 1:100 dilution. (B) IF microscopy of Mtb (H37Rv) with intact capsule showing reduced IgC binding with anti-AM Abdepleted sera compared with nondepleted sera. The brightness and contrast of the images were adjusted with the same settings using Image) (NIH). (C) Decreases in THP-1 phagocytosis of Mtb opsonized with nondepleted versus anti-AM Ab-depleted heat-inactivated sera (10\%). Columns and error bars represent mean and SD of triplicates (circles). ( $D$ and $\mathbf{E}$ ) Reduction of Mtb phagocytosis in relation to anti-AM IgC titer depletion for TST+IGRA ${ }^{+}$and TB sera. Sera were selected based on moderate to high anti-AM IgG titers (OD $>0.4$ for TST IGRA ${ }^{+}$subjects, and OD $>0.5$ for TB patients) and sufficient serum volume available to be able to assess the effects of reducing anti-AM Abs (TST+IGRA $\left.n=10, T_{B}^{+} n=13\right)$. Spearman's rank correlation. Duplicate or triplicate values are shown as circles. Results of assays repeated on separate days correlated highly and significantly $(r>0.8, P<0.0001)$.

We then performed antigen-specific Ab depletion studies on all sera with moderate to high anti-AM IgG titers from Mtb-infected subjects from whom we had sufficient sample available (10 $\mathrm{TST}^{+} \mathrm{IGRA}^{+}$and $13 \mathrm{~TB}$ sera). We assured a selective reduction of IgG reactivity to AM by demonstrating preservation of IgG reactivity to other Mtb surface or culture filtrate antigens such as the proteins GroEL2 and CFP10, respectively (Supplemental Figure 3). We then correlated the reduction of anti-AM IgG titer with functional changes in Mtb phagocytosis for the 2 phenotypically distinct groups with either controlled (TST' $\mathrm{IGRA}^{+}$) or uncontrolled Mtb infection (TB). Reduction in the anti-AM IgG titer correlated significantly with reduction in Mtb phagocytosis only for sera from $\mathrm{TST}^{+} \mathrm{IGRA}^{+}$subjects $(P=0.003$; Figure $4 \mathrm{D})$, not for sera from TB subjects $(P=0.29$; Figure $4 \mathrm{E}$ ), thereby demonstrating distinct functions of anti-AM IgG derived from $\mathrm{TST}^{+} \mathrm{IGRA}^{+}$compared with TB subjects.

Although slightly less effective than using AM-coupled beads, we achieved a similar anti-AM IgG reduction by incubating sera with Mtb bacteria with an intact capsule (H37Rv grown without detergent; Figure 5A). The corresponding reduction in Mtb phago- cytosis by THP-1 cells coincubated with sera depleted for Abs by this method was, although considerable in comparison with nondepleted sera, not quite as pronounced in all of the samples in comparison with sera depleted with AM-coupled Dynabeads (Figure 5B). It is conceivable that the observed differences in phagocytosis between depletion with whole bacteria and depletion with AM-coupled beads could be due to either incomplete depletion of anti-AM IgG, or reduction of other anti-Mtb surface Abs that influence interactions between the Mtb surface and host cell surface receptors when depleting with whole bacteria. These hypotheses warrant further investigation with various anti-Mtb surface mAbs - experiments that were beyond the scope of the current studies. To assure that our observed effects were not restricted to THP-1 cells, we validated the reduction in phagocytosis after depleting sera for Mtb surface-specific IgG with human PBMC-derived macrophages (Figure 5C).

Although exploration of the involvement of specific Fc $\gamma$ receptors (Fc $\gamma R s)$ was not a primary objective of this study, we further assessed the reduction in Mtb phagocytosis when THP-1 cells were 

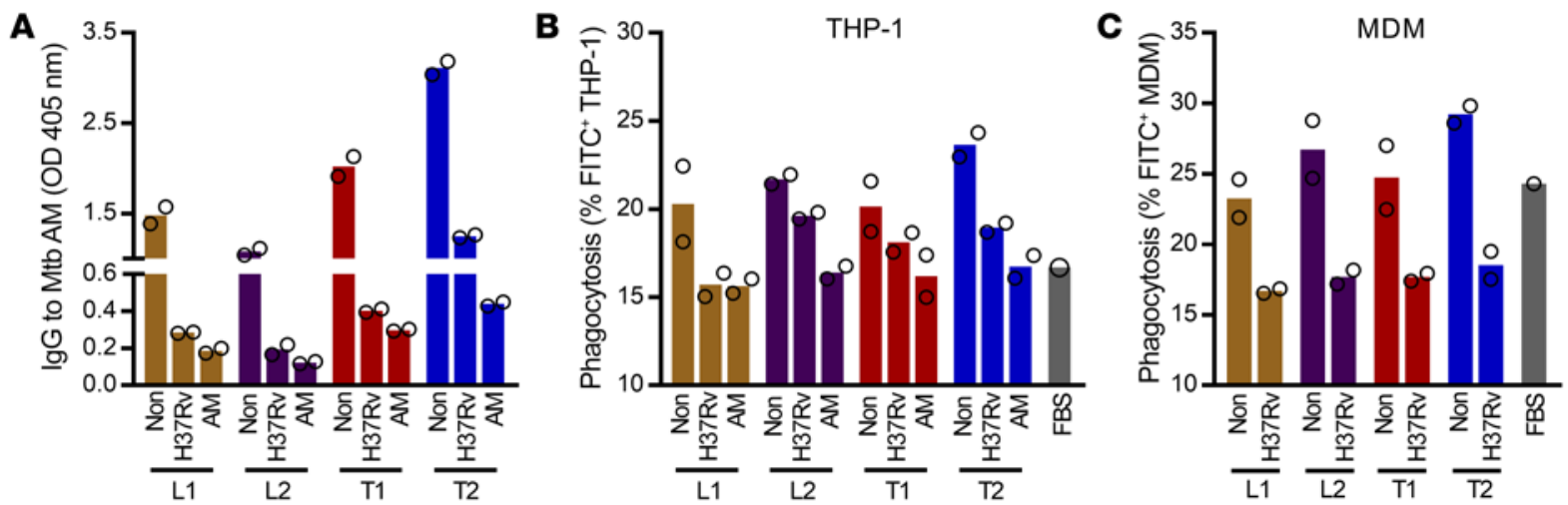

Figure 5. Coincubation of sera with Mtb with an intact capsule or AM-coupled beads reduces anti-AM IgC titers and Ab-mediated phagocytosis in THP-1 and human monocyte-derived macrophages. (A) Anti-AM IgG titers in heat-inactivated sera (1:100) before (Non) and after depletion of Abs against the Mtb surface (H37Rv, grown without detergent) or Mtb capsular AM (coupled to beads). Sera tested were from 2 TST+IGRA+ (L) and 2 TST $^{+}$IGRA $(T)$ subjects with high anti-AM IgC titers. No significant difference in anti-AM IgC titer reduction was observed between the 2 depletion methods ( $P=0.23$, paired $t$ test). (B) Comparison of Ab effects between nondepleted (Non) and anti-Mtb surface or anti-AM Ab-depleted sera (10\%) on Mtb phagocytosis by THP-1 cells. (C) Reduction of Mtb phagocytosis by human monocyte-derived macrophages (MDMs) coincubated with sera (10\%) depleted of Ab against the Mtb surface (H37Rv). Columns represent mean of duplicates (circles). Because of the limited availability of cells and the focus on comparing nondepleted with antigen-specific Ab-depleted sera, FBS was not tested in duplicates.

blocked with mAbs against Fc $\gamma$ Rs before and during coincubation with Mtb and sera from the 4 asymptomatic subjects with high anti-AM IgG titers ( $2 \mathrm{TST}^{+} \mathrm{IGRA}^{+}$and $\left.2 \mathrm{TST}^{+} \mathrm{IGRA}^{-}\right)$. We observed a significant reduction when blocking Fc $\gamma$ RI, which was even more pronounced when we blocked Fc $\gamma$ RII (Figure 6). These data are consistent with other studies indicating that enhanced Mtb phagocytosis by human anti-Mtb Abs is Fc $\gamma \mathrm{R}$ mediated $(17,19)$.

$M t b$ phagocytosis is associated with IgG reactivity to 5 AM OS motifs in TST $T^{+} I G R A^{+}$subjects. The anti-AM IgG titers among subject groups were not only greatly variable, they were also tremendously heterogeneous in their binding specificity to AM OS motifs within the PS (Figure 7A). Interestingly, while the overall anti-AM IgG titers were significantly higher in the TB compared with the $\mathrm{TST}^{+} \mathrm{IGRA}^{+}$group (Figure 1 ), $\mathrm{TST}^{+} \mathrm{IGRA}^{+}$ subjects recognized many AM OS motifs with the same intensity as TB subjects. Whereas there was no significant correlation for any of the 29 AM OSs in the TB group, Mtb phagocytosis was significantly associated with reactivity to 5 OSs $(3,7,8,9$, and 49) in $\mathrm{TST}^{+} \mathrm{IGRA}^{+}$(Figure 7B and Supplemental Table 1). In contrast to many other AM OS motifs included in our microarray (ref. 39 and Supplemental Table 1), these 5 AM OSs share a common motif of 4 Araf residues (Figure 7C, green stars) attached in the same configuration, $\beta-(1 \rightarrow 2), \alpha-(1 \rightarrow 5), \alpha-(1 \rightarrow 5)$, and further functionalized with additional mannose (green circles), inositol phosphate, or 5-thiomethyl-xylose (orange stars) residues. Notably, AM OS motifs 3, 6, and 12, whose IgG rec-
A

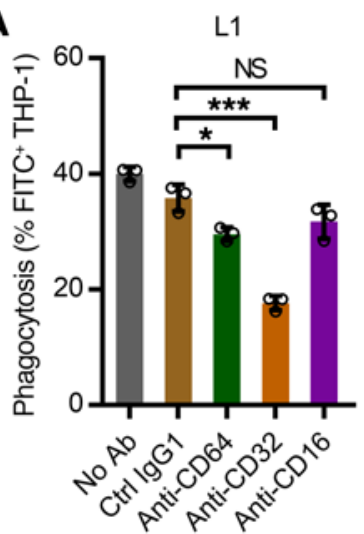

B

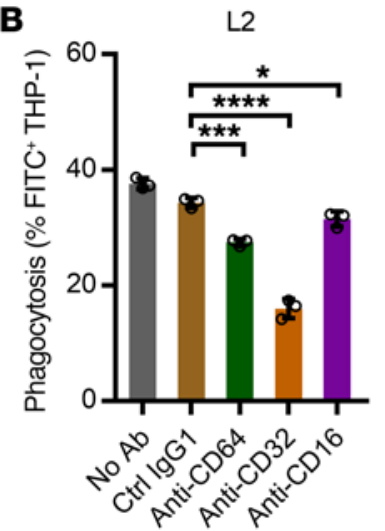

C

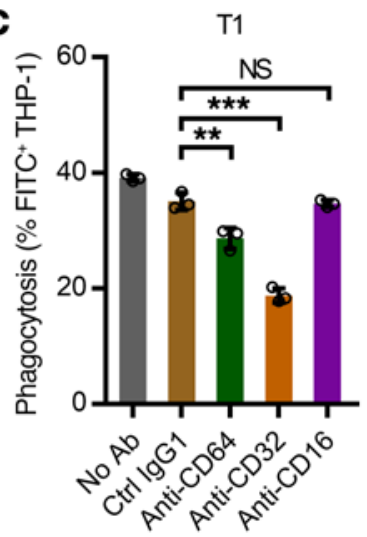

D

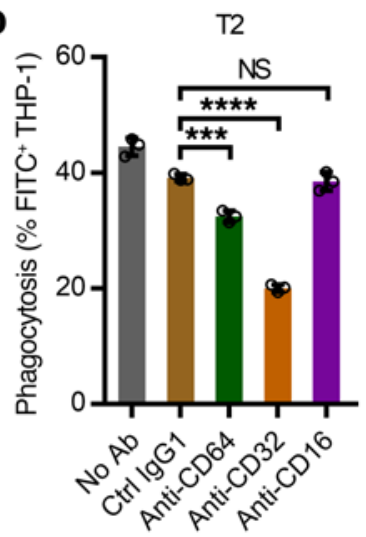

Figure 6. Reduction of Ab effects on Mtb phagocytosis when Fc $\gamma$ Rs were blocked. Anti-human Fc $\gamma \mathrm{R}$ murine mAbs or isotype-matched control murine mAb (Ctrl IgG1) were added to THP-1-derived macrophages before Mtb infection (H37Ra; MOI 20) and coincubation with 10\% heat-inactivated sera from 4 asymptomatic TST+IGRA ${ }^{+}(\mathrm{L})(\mathbf{A}$ and $\mathbf{B})$ or asymptomatic TST+IGRA ${ }^{-}(\mathrm{T})$ subjects (C and $\left.\mathbf{D}\right)$ with high anti-AM IgC titers. The blocking murine mAbs were anti-human CD64 (clone 10.1) against Fc $\gamma R$ I, anti-human CD32 (clone AT10) against Fc $\gamma$ RII, and anti-human CD16 (clone 3C8) against Fc $\gamma$ RIII. The phagocytosis was assessed at 3 hours after infection. Columns and error bars represent mean and SD of triplicates (circles). Representative data from 2 experiments. Unpaired $t$ test. ${ }^{*} P<0.05,{ }^{* *} P<0.01,{ }^{* *} P<0.001$, ${ }^{* * *} P<0.0001$; NS, not significant $(P \geq 0.05)$. 
A $\quad$ TST $^{+}$IGRA $^{+}$

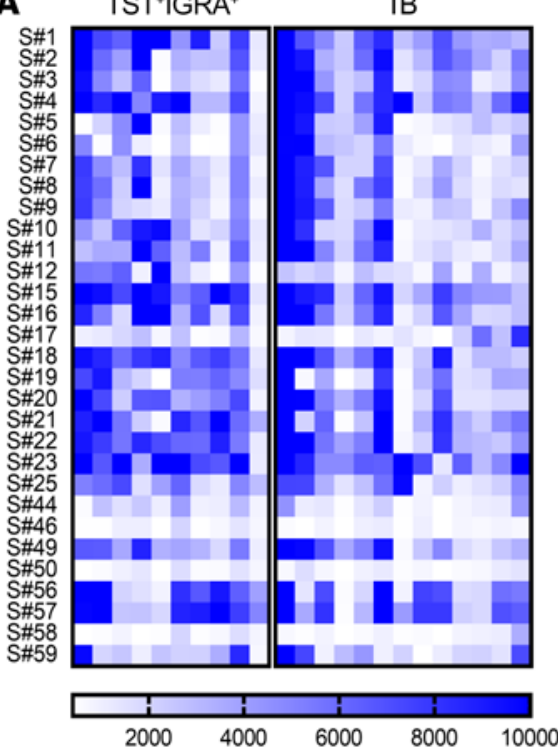

B

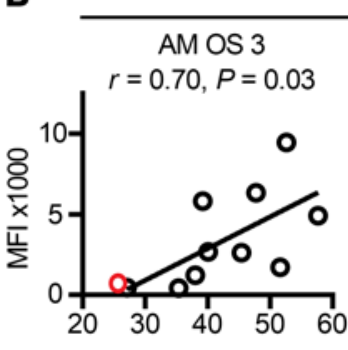

$\mathrm{TST}^{+}$IGRA ${ }^{+}$

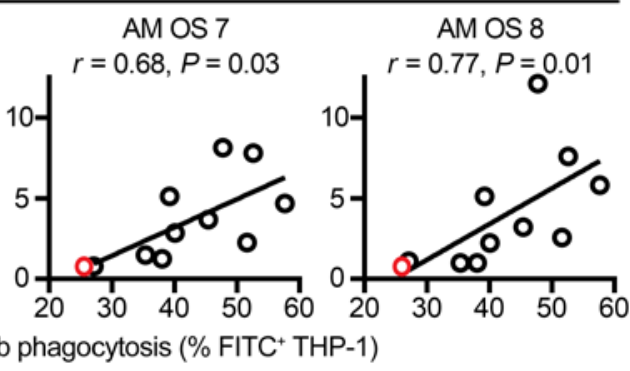

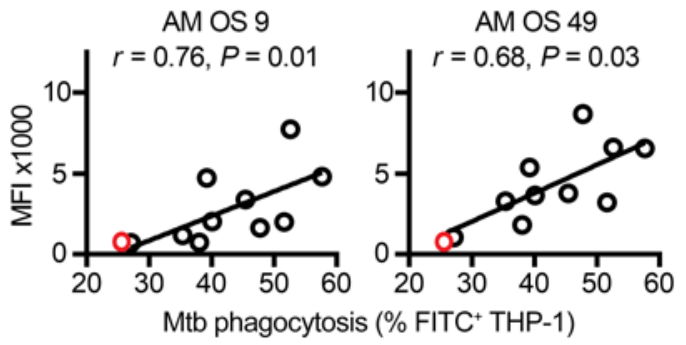
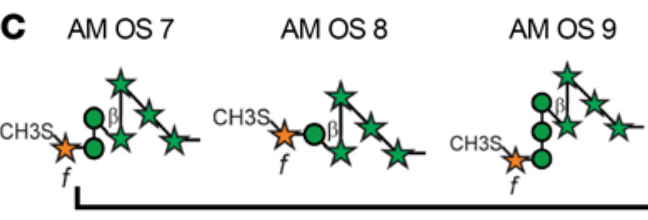

AM OS 49

AMOS $3 \quad$ AMOS 6

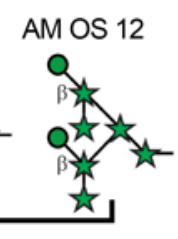

$\mathrm{TST}^{+}$IGRA ${ }^{+}$

BCG vaccination

Figure 7. Significant correlations between IgG reactivity to specific AM OS motifs and Ab-mediated Mtb phagocytosis with TST+IGRA+ but not TB sera. (A) Heterogeneous serum IgG reactivity to AM OS in both TST+IGRA ${ }^{+}$and TB groups. Color features represent the mean fluorescence intensity (MFI) to each of the OS motifs. TST+IGRA ${ }^{+}$subjects recognized many AM OS motifs with the same intensity as TB subjects (10\% of color features in the TST'ICRA ${ }^{+}$ group and $9 \%$ of color features in the TB group exceeded the upper scale limit). Sera lined from left to right had IgG reactivity to native AM from high to low (minimum OD > 0.4). Compound S\#46 is an OS motif of the mycobacterial capsular PS $\alpha$-glucan, which served as a control. (B) Significant correlations between IgG reactivity to AM OS and Mtb phagocytosis were seen for 5 OSs with TST+IGRA+ $(n=10)$ but none of the 30 OSs with TB sera ( $n=13$; see also Supplemental Table 1). Spearman's rank correlation. Red circles, TST- control serum. (C) The 5 AM OSs recognized by TST+IGRA ${ }^{+}$sera and significantly associated with Mtb phagocytosis share a common motif of 4 Araf residues (green stars) attached in the same configuration, $\beta-(1 \rightarrow 2), \alpha-(1 \rightarrow 5), \alpha-(1 \rightarrow 5)$, and further functionalized with additional mannose (green circles), inositol phosphate, or xylose (orange stars) residues. IgG reactivity to AM OSs 3, 6, and 12 also correlated with BCG phagocytosis in our prior studies with BCG immune sera (16). OS 3 recognition is significantly associated with phagocytosis with both TST+IGRA ${ }^{+}$and BCG sera, while significant association with the other 2 motifs ( 6 and 12) was only seen with BCG immune sera.

ognition correlated significantly with $\mathrm{Ab}$ functions in our prior studies with BCG immune sera, all have a similar core motif (ref. 16 and Figure 7C).

Anti-AM IgG from high-titer asymptomatic BCG-vaccinated and/or Mtb-exposed/infected subjects, but not TB patients, reduces $M t b$ intracellular growth. To study the effects of anti-AM Ab on mycobacterial intracellular growth, THP-1-derived macrophages were coincubated with high-titer sera in nondepleted and antiAM Ab-depleted states. Because $\mathrm{TST}^{+} \mathrm{IGRA}^{+}$subjects had significantly lower anti-AM IgG responses than TB patients, we selected only those samples that had an anti-AM IgG titer reduction above 0.5 OD when depleted with AM-coupled beads. Of the 5 samples meeting this criterion (Figure 4D), we had sufficient remaining volume available for 4 . We further included the few high-titer sera from the $\mathrm{TST}^{+} \mathrm{IGRA}^{-}$group and the $\mathrm{TST}^{-}$group that met this criterion and for which we also had sufficient volume available for depletion and growth reduction experiments $(n=3)$. Because we observed substantial Mtb killing, irrespective of anti-AM Ab depletion, with sera obtained from TB patients already started for a few days on antituberculous treatment, we included only TB samples that were obtained before the treatment initiation (available for 5 pulmonary TB patients). Consistent with our observation of differences in anti-AM IgG-mediated Mtb phagocytosis between $\mathrm{TST}^{+} \mathrm{IGRA}^{+}$and $\mathrm{TB}$, we found that intracellular Mtb growth rates were significantly lower in macrophages coincubated with nondepleted compared with anti-AM Ab-depleted sera from $\mathrm{TST}^{+} \mathrm{IGRA}^{+}$, $\mathrm{TST}^{+} \mathrm{IGRA}^{-}$, or $\mathrm{TST}^{-}$asymptomatic subjects with high anti-AM IgG titers (Figure 8A). By contrast, high-titer nondepleted compared with anti-AM Ab-depleted sera from TB patients (before antituberculous therapy) enhanced Mtb intracellular growth (Figure 8B). Sera depleted with control (BSA-coupled) beads had no effect on Mtb intracellular growth compared with nondepleted sera (Supplemental Figure 4). When testing the nondepleted serum from the asymptomatic subject showing the highest inhibitory effects on Mtb intracellular growth, we found enhanced phagosome-lysosomal fusion in Mtb-infected macrophages (Figure $8 \mathrm{C}$ ), consistent with results with BCG immune sera with high anti-AM IgG titers (16). 
A

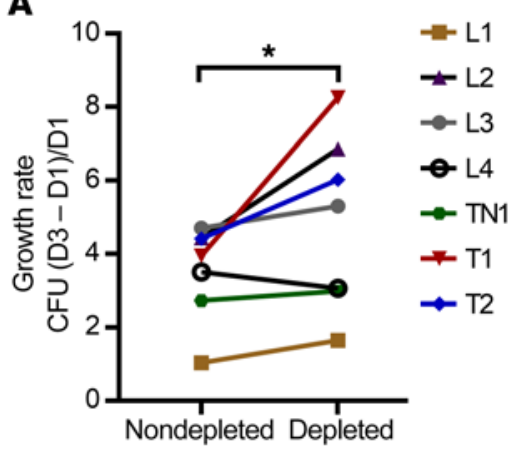

B

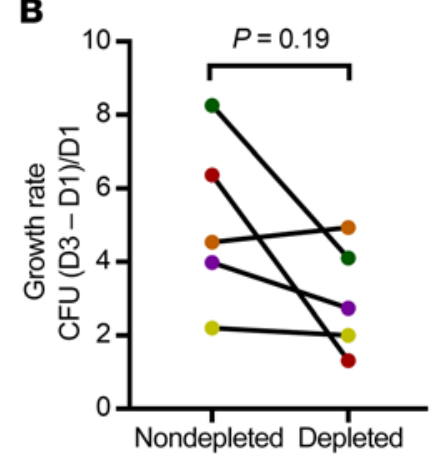

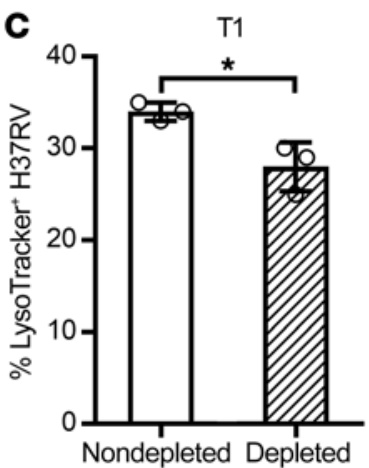

Figure 8. Protective in vitro efficacy seen with anti-AM Abs from asymptomatic individuals with BCG or Mtb exposure/infection but not with anti-AM Abs from TB patients. Effects of nondepleted versus anti-AM Ab-depleted sera on intracellular Mtb growth (H37Rv; MOI 1) in human macrophages (THP-1), indicating that anti-AM Abs from asymptomatic subjects reduce while those from TB patients enhance intracellular Mtb growth. Experiment repeated with selected sera showed the same results. Sera depleted with control (BSA-coupled) beads had no effects on Mtb intracellular growth compared with nondepleted sera (Supplemental Figure 4). (A) Differences in intracellular growth rates with sera from TST+IGRA ${ }^{+}(\mathrm{L}, n=4)$ and TST+IGRA $\left(\mathrm{T}^{+}, n=2\right)$ or TST- $\left(\mathrm{TN}^{+}, n\right.$ $=1$ ) subjects with the highest anti-AM IgG titers. Wilcoxon matched-pairs signed-rank test. (B) Differences in intracellular growth rates with sera from TB patients with high anti-AM IgC titers ( $n=5$; all pulmonary TB). Note that our power was limited because only 5 high-titer sera with sufficient volume were available before the start of antituberculous therapy. (C) High-anti-AM IgG serum (T1) enhanced phagolysosomal fusion in Mtb-infected THP-1 cells (MOI 5) significantly more than anti-AM Ab-depleted serum. Columns and error bars represent mean and SD of triplicates (open circles). Unpaired $t$ test. ${ }^{*} P<0.05$.

Passive transfer of human anti-AM-specific polyclonal IgG from high-titer asymptomatic TST IGRA and TST $T^{+} I G R A^{+}$subjects shows protective efficacy in Mtb-infected mice. Of the serum samples with high anti-AM IgG titers for which we were able to show protective in vitro effects against Mtb (Figure 4, A-C, and Figure 8A), we had sufficient serum volume available from 4 asymptomatic subjects $\left(2 \mathrm{TST}^{+} \mathrm{IGRA}^{+}\right.$and $\left.2 \mathrm{TST}^{+} \mathrm{IGRA}^{-}\right)$to isolate anti-AM polyclonal IgG. Notably, the 4 subjects had different levels of IgG subclass reactivity to AM. IgG1 and IgG2 were the predominant subclasses in 3 subjects, and 1 subject with $\mathrm{TST}^{+} \mathrm{IGRA}^{+}$(L2) had an almost exclusive anti-AM IgG2 reactivity (Figure 9A). We first ensured that the purified antiAM-specific polyclonal IgG (a) had higher affinity to native AM compared with the corresponding total serum IgG at the same concentration (Supplemental Figure 5A), (b) recognized AM OS compounds in a pattern similar to that seen with the anti-AM Ab-nondepleted serum (Supplemental Figure 5B), and (c) bound to the surface of Mtb with an intact capsule (Figure 9B). Whereas all donors had IgG reactivity to a range of AM OS motifs, each donor reacted strongly with at least 2 of the $5 \mathrm{AM}$ OS motifs associated with IgG functions in vitro. We then validated the in vitro function of the purified anti-AM IgG, showing enhanced Mtb phagocytosis by THP-1-derived macrophages when coincubated with $10 \mu \mathrm{g} / \mathrm{mL}$ to as low as $1 \mu \mathrm{g} / \mathrm{mL}$ of the subjects' anti-AM polyclonal IgG (Figure 9C).

To evaluate protective in vivo efficacy, mice (C57BL/6) were injected with $8 \mu \mathrm{g}$ anti-AM IgG i.p. 1 day before and $4 \mu \mathrm{g}$ i.p. 1 day after low-dose Mtb aerosol infection ( 10 CFU Erdman; Figure 9D). We used Mtb Erdman for in vivo experiments because this strain is more virulent and grows faster than $\mathrm{H} 37 \mathrm{Rv}$ (40). Because the i.p. administration of $\mathrm{Ab}$ in mice equilibrates between intravascular and other body compartments within 24 hours, and then slowly declines (41), the $\mathrm{Ab}$ dose before and after infection was chosen to simulate the approximate calculated amount of anti-AM IgG in the high-titer human subjects. Human Abs are not detectable in mice after 7-10 days (19). Therefore, and because purified polyclonal anti-AM $\operatorname{IgG}$ was only available in limited amounts, animals were euthanized at 2 weeks after Mtb infection. Because of the limited amount of anti-AM IgG, we could test only 5 mice per treatment group. Unfortunately, in the normal saline (NS) control group, 1 mouse died for unknown reasons, and the lung of another mouse was contaminated with Proteus mirabilis, which can occasionally happen (42). Despite this constraint in statistical power and the very low dose of polyclonal anti-AM used, we could show protective efficacy of anti-AM IgG. Compared with the control mice injected with NS, mice treated with anti-AM IgG from 2 TST $^{+}$IGRA ${ }^{-}$subjects had lower lung CFU $(P=0.050$ and 0.074$)$, and mice treated with anti-AM IgG from $1 \mathrm{TST}^{+} \mathrm{IGRA}^{+}$subject with an almost exclusive anti-AM IgG2 response had significantly lower lung CFU at 2 weeks after infection $(P=0.036$; Figure 9D). Passive transfer from 1 patient with pulmonary TB, for whom we had sufficient serum volume for isolation of anti-AM-specific IgG available, did not show any protective efficacy in a separately performed experiment (Supplemental Figure 6). These in vivo data provide further evidence for the protective role of human anti-AM-specific IgG isolated from some BCG-vaccinated and/or Mtb-exposed/ infected asymptomatic subjects.

\section{Discussion}

Despite the increasing evidence for a role of Ab-mediated immunity against TB in humans, knowledge of the most relevant mycobacterial antigens and epitopes remains limited. Prior human and murine studies have supported targeting the mycobacterial surface PS AM (11, 31-34). However, human data were based on associations of anti-AM IgG titers with functions against BCG, and murine studies were limited in capturing the heterogeneity of Abs elicited with vaccination and during natural Mtb infection in humans. Here we show that in natural Mtb infection human IgG and subclass responses to Mtb capsular AM are not only highly variable but also tremendously heterogeneous in their reactivity to OS motifs within AM. In addition, we have compared the in vitro effects of sera with moderate to high anti-AM IgG titers to the 
A
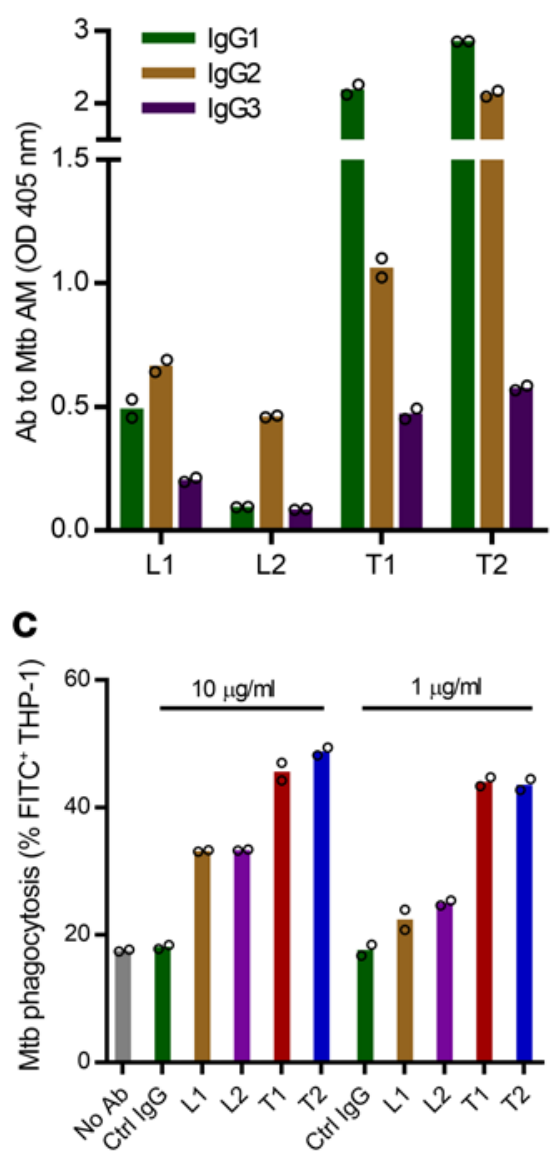

B

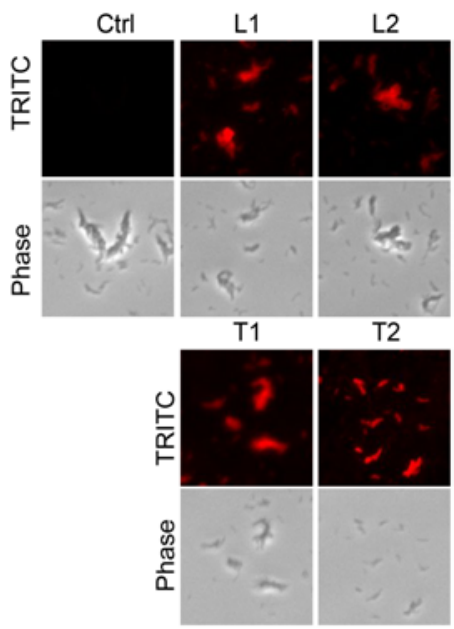

D

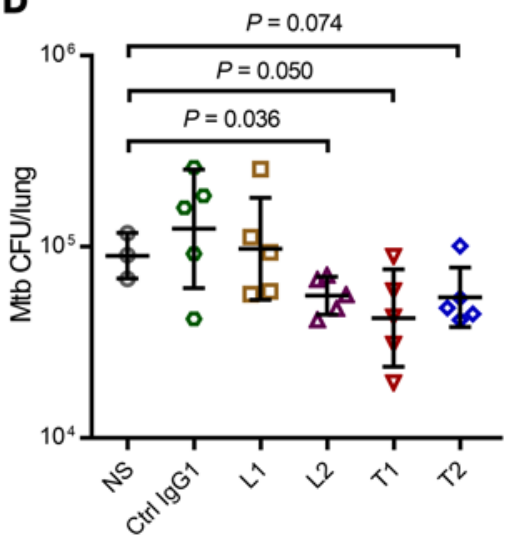

Figure 9. Passive transfer of purified human antiAM polyclonal IgG from TST ${ }^{+}$IGRA ${ }^{+}$and TST $^{+}$IGRA- asymptomatic subjects reduces mycobacterial burden in Mtb-infected mice. (A) Serum IgG subclass reactivity to H37Rv AM by ELISA from 2 TST $^{+}$IGRA $^{+}$ ( $\mathrm{L} 1$ and $\mathrm{L} 2$ ) and $2 \mathrm{TST}^{+} \mathrm{IGRA} \mathrm{A}^{-}$subjects (T1 and T2). Columns represent mean of duplicates (circles). (B) Purified anti-AM polyclonal IgG bound to the Mtb surface (H37Rv grown without detergent to preserve the capsule) by IF microscopy (see Supplemental Figure 5 for further antigen binding data of purified anti-AM IgG). Ctrl, total IgG purified from 1 TST $^{-}$US-born subject with very low anti-AM IgC titer (same for Ctrl $\operatorname{IgG}$ in C). (C) Purified anti-AM polyclonal IgG enhanced Mtb phagocytosis by THP-1 cells coincubated with 10 or $1 \mu \mathrm{g} / \mathrm{mL}$ Ab. Columns represent mean of duplicates (circles). (D) Mice (C57BL/6) received NS, $8 \mu \mathrm{g}$ irrelevant IgG1 control mAb, or $8 \mu \mathrm{g}$ purified polyclonal anti-AM IgC i.p. 24 hours before and NS, $4 \mu \mathrm{g}$ control, or $4 \mu \mathrm{g}$ anti-AM IgG i.p. 24 hours after aerosolized low-dose Mtb infection (Erdman; mean lung CFU $11 \pm$ 5 one day after infection). Data show lung CFU (mean and SD) 2 weeks after infection. One-way ANOVA, $P$ $=0.025 . T$ test without $(\mathrm{L} 2)$ and with $(\mathrm{T} 1, \mathrm{~T} 2)$ Welch's correction for unequal variances. effects of anti-AM Ab-depleted sera on opsonophagocytosis and intracellular Mtb growth. These studies reveal that the polyclonal anti-AM IgG derived from asymptomatic subjects (BCG vaccinated, Mtb exposed, and LTBI), in contrast to TB, has protective efficacy. In line with data from our work with human BCG immune sera (16), we show that in controlled (but not uncontrolled) Mtb infection the opsonophagocytic activity correlated significantly with $\operatorname{IgG}$ reactivity to specific OS motifs within the PS. We further demonstrate that passive transfer of very low doses of purified polyclonal anti-AM IgG $(12 \mu \mathrm{g})$, isolated from a few asymptomatic high-titer subjects with protective anti-AM in vitro functions, has protective in vivo efficacy against Mtb in the low-dose murine TB model. Interestingly, although limited to correlative in vitro data and protective in vivo data with passive transfer of anti-AM IgG from 1 asymptomatic $\mathrm{TST}^{+} \mathrm{IGRA}^{+}$subject with an almost exclusive anti-AM IgG2 response, our data also suggest a potential role of anti-AM IgG2. Although further studies are needed to determine both the relevance of specific anti-AM $\mathrm{Ab}$ features and other potentially protective Mtb antigens, our data demonstrate a key role of AM in Ab-meditated immunity against TB in humans and suggest the importance of targeting specific glycan epitopes.

The capsule of Mtb is a critical virulence factor and consists mainly of PS, of which AM is an important immunogenic component $(23-25,33)$. We show that binding of human polyclonal IgG to Mtb with an intact capsule is considerably reduced when it is depleted of AM-specific Abs. Because we worked with Mtb labo- ratory strains and the capsule composition and its AM accessibility could differ between Mtb strains (33), we ensured that the reactivity of $\operatorname{IgG}$ from all groups correlated highly and significantly to capsular AM isolated from laboratory versus clinical Mtb strains. Several mechanisms could explain our observations. Capsular PSs aid bacteria, including Mtb, in invading host cells by inhibiting host defense mechanisms such as macrophage phagocytosis and intracellular killing $(24,43)$. However, Mtb surface glycans, by interacting with macrophage surface receptors, can also mediate adhesion and internalization (23). For example, LAM promotes adhesion to macrophages and uptake via interaction with mannose receptors (44). This cell entry mechanism allows intracellular survival and replication of Mtb by limiting phagosome-lysosomal fusion, a mechanism that can be blocked by a murine mAb against LAM (44). Furthermore, a key mechanism for Ab-mediated effects against intracellular pathogens, including BCG, is enhancement of lysosomal degradation of pathogens via Fc $\gamma \mathrm{R}$ signaling, a mechanism independent of Fc $\gamma \mathrm{R}$-mediated phagocytosis (45). It is thus conceivable that the protective effects of anti-AM IgG seen in our studies are based on both preventing host cell adhesion and Mtb macrophage uptake through mannose or other host receptors, and simultaneously promoting Fc $\gamma$ R-mediated phagocytosis and intracellular growth inhibition via Fc $\gamma \mathrm{R}$ signaling.

Remarkably, depletion of anti-AM IgG from high-titer TB sera did not correlate with the reduction of Mtb phagocytosis. Moreover, Mtb intracellular growth in vitro was enhanced in the pres- 
ence of anti-AM IgG from TB patients. Because of the high serum volume required, we were able to isolate sufficient anti-AM IgG from only 1 high-titer pulmonary TB patient for evaluation in vivo, limiting both our ability to assess whether the macrophage growth data were a good in vitro correlate of in vivo effects and our ability to test the protective in vivo efficacy of TB patient-derived antiAM IgG. Mice receiving, before and after Mtb infection, anti-AM IgG isolated from this TB patient had no significant difference in Mtb lung burden compared with control mice. Our findings of protective Abs in asymptomatic Mtb-exposed or -infected individuals are in line with results from 2 other recent studies $(17,19)$. Although these studies did not investigate antigen-specific Abs, $\mathrm{Lu}$ et al. found human serum IgG against purified protein derivative (PPD) from LTBI but not TB patients to have protective Ab functions against Mtb in vitro (17), and Li et al. showed that passive transfer of total IgG from some asymptomatic Mtb-exposed or -infected health care workers (but not TB patients) has protective in vivo efficacy in Mtb-infected mice (19). In contrast, investigating mycobacterial growth in an ex vivo mycobacterial growth inhibition assay (MGIA), O'Shea et al. found that whole blood from individuals with TB controlled BCG (Pasteur) growth better than that from individuals with LTBI. However, they did not observe a difference in control of growth of Mtb (H37Rv) (46). Although Lu et al. found an association of the protective $\operatorname{IgG}$ in vitro functions with distinct glycosylation profiles in the IgG Fc region (17), Li et al. found a reversal of protective ex vivo effects in a whole-blood assay when preabsorbing IgG from protective donors against heatkilled Mtb but not soluble Mtb antigens (19). Direct comparisons with our study results are limited because of the different experimental methods and the use of whole blood, total IgG, or IgG against PPD (which contains 100-200 varying denatured Mtb proteins and moderate amounts of LAM; ref. 47). Nevertheless, these findings in aggregate suggest that multiple components of the humoral immune response at different stages of Mtb infection play a role in the defense against Mtb.

Because of their important role in adhering to and invading host cells, pathogen surface glycans are targeted by various vaccines (reviewed in refs. 20-22). However, PSs are complex molecules that include determinants that can elicit a functionally diverse spectrum of Abs, including protective, nonprotective, or even disease-enhancing Abs (20-22). Using recently developed glycan arrays with synthetically generated fragments of AM OS motifs (39), we found that the anti-AM IgG response elicited in Mtb infection is highly heterogeneous in its reactivity to AM OS motifs within the PS. Congruent with our prior studies with BCG immune sera (16), our current results suggest that the protective functions seen with BCG immune and $\mathrm{TST}^{+} \mathrm{IGRA}^{+}$sera are associated with IgG reactivity to certain AM glycan epitopes. These fragments all share a common motif of 4 Araf residues attached in the same configuration, and further functionalized with additional residues - mannose, inositol phosphate, or 5-thiomethyl-xylose. We further note that these motifs are all found at the terminal end of AM, and their structures differ considerably from inner motifs (26). Although these data are correlative, they provide important information for the generation of OS-specific mAbs. Such mAbs would have defined binding specificities that would allow the detailed delineation of functions and mechanisms against
OS-specific AM epitopes. The significance of the structural similarities of AM motifs associated with IgG functions in humans is unknown at present. Our data indicate that Abs targeting the terminal motifs of AM could interfere differently in host-pathogen interactions compared with Abs targeting the inner motifs of capsular AM. These data could have important potential implications because the field of glycoconjugate vaccine development is moving to the use of OS-conjugate vaccines that have the advantage of both greater product consistency and induction of Abs against the most relevant glycan epitopes (20-22).

In addition to the antigen-binding variable region, $\mathrm{Ab}$ effector functions are influenced by the subclass and the glycosylation pattern in the IgG Fc region (reviewed in refs. 13, 14, 48). Although some functions of human $\mathrm{Ab}$ isotypes against $\mathrm{Mtb}$ (e.g., IgA against heparin-binding hemagglutinin [HBHA]) have recently been established (18), less is known about antigen-specific IgG subclass functions. In their whole-blood MGIA results, O'Shea et al. found a weak but significant correlation between IgG1 responses to ESAT-6/CFP10 and growth reduction of Mtb (46). Lu et al. did not find differences in IgG subclass responses to PPD between LTBI and TB patients (17), whereas Li et al. found, despite no overall IgG3 differences, higher human IgG3 levels in mice after the first days of passive transfer with protective versus nonprotective IgG (19). During natural bacterial infection in humans, PSs typically induce IgG2 responses, while IgG1 is readily induced by glycoconjugate vaccines (48). Consistent with our prior data $(16,30)$, we found high IgG1 and IgG2 but little IgG3 or IgG4 reactivity to AM in both BCG-vaccinated and Mtb-infected subjects regardless of Mtb infection stage. Interestingly, when we compared anti-AM IgG2/IgG1 subclass ratios, individuals with LTBI had a significantly higher ratio than TB patients. In a subgroup analysis comparing IgG and subclass responses to AM in patients with localized pulmonary versus disseminated TB, we further found that patients with disseminated TB had lower anti-AM IgG titers and that this difference was driven by significantly lower anti-AM IgG2 compared with IgG1. The lack of difference in anti-AM IgG1 responses between localized and disseminated TB suggests that the difference in anti-AM subclass reactivity is unlikely to be driven by immune complex formation. Our data are further in line with a study by Costello et al., who, although not investigating subclasses, found significantly lower anti-LAM IgG responses, independent of immune complex formation, in young children with disseminated compared with those with localized TB (49). Findings from a recent study by Logan et al. support a potential role of IgG2 in Mtb infection (50). Although not investigating antigen-specific responses, the authors found that BCG-vaccinated infants who remained Mtb uninfected in a highly TB-endemic region had significantly higher IgG2 responses to BCG than those who, over a 2-year follow-up, became Mtb infected. Although human IgG1 has favorable properties in Ab-mediated immunity, as it binds complement and all Fc $\gamma$ Rs (reviewed in refs. 13, 14, 48), human IgG2 is generally believed to have decreased ability to activate complement and engages predominantly Fc $\gamma$ RII $(13,14,48)$. However, IgG2 can be highly active in the presence of complement and at high epitope concentrations on target cells, such as PS antigens on encapsulated bacteria (51). It is thus not surprising that children and adults with recurrent infections with encapsulated bac- 
teria are frequently found to have $\operatorname{IgG} 2$ deficiency $(52,53)$. Consistently, passive transfer of human IgG subclass mAbs with the same variable region against glucuronoxylomannan, a capsular PS of the facultative intracellular fungus Cryptococcus neoformans, showed that IgG2 but not IgG1 protected mice against infection (54). We observed a strong correlation between anti-AM IgG2, but not IgG1, responses and Mtb phagocytosis in the asymptomatic $\mathrm{TST}^{+} \mathrm{IGRA}^{+}$group and the most pronounced reduction of phagocytosis when blocking Fc $\gamma$ RII. Furthermore, although limited to one $\mathrm{TST}^{+} \mathrm{IGRA}^{+}$subject, we show protective in vitro and in vivo efficacy with an almost exclusive polyclonal anti-AM IgG2 sample. Although these data are limited and require further investigation, they nevertheless, collectively with data from other human studies, indicate that both isotype and IgG subclass could play distinct roles in TB, depending on the antigens/epitopes targeted. Further studies, ideally with mAbs consisting of the same variable but different $\mathrm{Fc}$ regions, are needed to better understand the functions of IgG subclasses in TB pathogenesis.

Although the involvement of specific Fc $\gamma$ Rs was not a primary focus of this study, we show that the enhanced Mtb phagocytosis seen with our high-anti-AM-titer asymptomatic subjects was Fc $\gamma \mathrm{R}$ mediated. In contrast to Lu et al., who found that the increased functionality of polyclonal LTBI IgG against PPD was associated with certain Fc glycosylation patterns and enhanced binding to Fc $\gamma$ RIII (17), we observed the strongest reduction in Mtb phagocytosis when blocking Fc $\gamma$ RII. Determining the anti-AM IgG Fc glycosylation, an important feature that can influence Fc $\gamma$ R-mediated effects $(13,14,48)$, was not feasible in our study because of the limited quantity of anti-AM IgG. Furthermore, in order to not introduce another component of heterogeneity such as polymorphism of $\mathrm{F} c \gamma \mathrm{R}$ and other factors when working with PBMCs (reviewed in refs. 14, 38), we performed experiments predominantly with THP-1 cell line-derived human macrophages. These express all $\mathrm{Fc} \gamma \mathrm{Rs}$ and are a valuable tool to determine phagocytic activity of clinical Ab samples (55). However, they do not express all Fc $\gamma$ Rs in the same proportions as PBMC-derived macrophages (56). Therefore, it was not feasible to determine whether the predominance of Fc $\gamma$ RII-mediated phagocytosis was influenced by the higher expression of Fc $\gamma$ RII and lower expression of Fc $\gamma$ RIII on THP-1 compared with PBMC-derived macrophages $(55,56)$, due to Fc glycosylation features or due to the high titers of anti-AM IgG2, which engages Fc $\gamma$ RII. However, we are confident that our results are not restricted to THP- 1 cells, because, consistent with our prior studies (16), we show similar Ab-mediated Mtb phagocytosis enhancement as well as reduction with anti-AM $\mathrm{Ab}$-depleted sera between THP-1 and human PBMC-derived macrophages.

Systemic administration of a very low dose of AM-specific IgG, equivalent to levels found in our protective donors, to mice before low-dose aerosolized Mtb infection provided evidence for the protective in vivo efficacy of anti-AM IgG. We aimed to mimic the human natural infection dose as closely as possible (using 10 CFU) while assuring that all mice were infected. The limited quantity in anti-AM IgG restricted our statistical power. Nevertheless, we were able to show that, compared with controls, mice treated with anti-AM IgG from 3 of 4 asymptomatic high-titer subjects had lower lung CFU. Whereas all donors had IgG reactivity to a range of AM OS motifs, each donor reacted strongly with at least 2 of the
5 AM OS motifs associated with IgG functions in vitro. These in vivo data support the protective role of human IgG targeting the Mtb capsular PS AM.

Although it is critically important to identify immune correlates against Mtb in humans, the polyclonal nature and heterogeneity of $\mathrm{Ab}$ responses to Mtb antigens in humans also led to several limitations in our study. Based on prior data by us and others, we focused on IgG against capsular AM. The exploration of other potentially protective Mtb antigens, which might even work synergistically with the targeting of AM glycan epitopes, was beyond the scope of this study. Furthermore, restricted IgG quantities limited analysis of specific $\mathrm{Ab}$ features and mechanisms. We further note that although macrophages are the main site for infection and survival (37), Mtb is known to infect several mononuclear phagocytes in vitro and in vivo (57). However, exploring $\mathrm{Ab}$ functions with other phagocytes, as well as assessing the potential interactions of human Abs with other immune cells such as T cells, was beyond the scope of the current study. Nevertheless, we note that cell-mediated and Ab-mediated immunity likely work synergistically given their many interactions, including those with innate immunity. Evidence for this comes from our recent murine immunization studies showing that the induction of both is superior to either alone in the protection against TB, as well as from other human studies indicating important interactions between both arms of adaptive immunity $(11,19,46,58)$.

In conclusion, we demonstrate a key role of capsular AM in human Ab-mediated efficacy against Mtb. We show that anti-AM IgG derived from asymptomatic subjects (BCG vaccinated, Mtb exposed, and LTBI), in contrast to TB patients, has protective efficacy and is associated with reactivity to specific OS motifs within AM. Our data further suggest a role for anti-AM IgG2. We observed considerable heterogeneity in human anti-AM IgG titers, subclass responses, and reactivity to AM glycan epitopes at various stages of Mtb infection, suggesting the contribution of human anti-AM IgG to susceptibility versus protection against infection and TB development in humans. A better understanding of the relevance of subclass and AM glycan epitopes will now require larger validation studies and investigations with anti-AM mAb variants in various in vitro and animal models, including Fc $\gamma$ R-humanized mice and nonhuman primates. Overall, our findings are of potential high significance because they suggest that, in addition to cellmediated responses, eliciting Abs against specific Mtb capsular glycan epitopes may increase vaccine efficacy against TB.

\section{Methods}

Subjects. Already collected samples from subjects enrolled in a cross-sectional TB immunodiagnostic study from in- and outpatient settings of 4 public hospitals in New York City from 2012 to 2016 were analyzed. Asymptomatic healthy volunteers without known risk factors for HIV infection were categorized by TST and whole-blood IGRA (QuantiFERON-TB Gold, Qiagen). In our institutions, TST is routinely performed, and, consistent with the American Thoracic Society/Infectious Diseases Society of America recommendations, in the absence of recent Mtb exposure history, a second diagnostic test (IGRA) was performed when the initial test (TST) was positive (36). Although an asymptomatic subject was considered latently Mtb infected if both tests were positive, a $\mathrm{TST}^{+} \mathrm{IGRA}^{-}$subject could have still been infect- 
ed if the risk of remote Mtb exposure was high, such as in immigrants from TB-endemic regions (36). We therefore categorized asymptomatic subjects into 3 groups: $\mathrm{TST}^{-}$, $\mathrm{TST}^{+} \mathrm{IGRA}^{-}$, and $\mathrm{TST}^{+} \mathrm{IGRA}^{+}$. Patients with TB were HIV uninfected, Mtb culture confirmed, and enrolled within 7 days of TB diagnosis. Based on symptoms and results from physical exams, radiographic imaging, and diagnostic work-up, TB patients were further categorized as having localized pulmonary or disseminated TB (defined as evidence for extrapulmonary TB and/or miliary $\mathrm{TB}$ on radiographic imaging).

Mtb strains. Mtb H37Ra, H37Rv, Erdman, CDC1551, and BCG (Pasteur) were grown in Middlebrook 7H9 medium supplemented with $10 \%$ (vol/vol) oleic albumin dextrose catalase (OADC) enrichment, $0.5 \%$ (vol/vol) glycerol, and with or without $0.05 \%$ tyloxapol or Tween-80. To generate AM, bacteria were subcultured into minimal media (11). Since we found no differences between phagocytosis rates using the avirulent H37Ra or the virulent H37Rv Mtb strain, H37Ra, allowing for biosafety level 2 (BSL-2) conditions, was used for phagocytosis experiments. Because of differences between H37Ra and H37Rv in intracellular growth (59), we used H37Rv under BSL-3 conditions for growth inhibition assays and Erdman for murine infection.

Mycobacterial antigen. Capsular AM was isolated and purified from the Mtb strains as previously described (11). Briefly, to preserve the capsule formation, mycobacteria were incubated in minimal media in the absence of the detergents (tyloxapol or Tween-80), and grown stationary at $37^{\circ} \mathrm{C}$ for 21 days in horizontally placed roller bottles. The cells were then harvested, treated with glass beads, resuspended, and centrifuged. The collected supernatants were filtered through 0.22 $\mu \mathrm{m}$, and the capsular antigen extracts isolated and purified by column chromatography and then lyophilized. Regarding our control antigens, we expressed the mycobacterial surface protein GroEL2 as recombinant histidine-tagged protein in Mycobacterium smegmatis, and purified it using Ni-NTA agarose (Qiagen). The recombinant culture filtrate protein CFP10, expressed in E. coli, was purchased from the Biodefense and Emerging Infections Research Resources Repository (BEI Resources; NR-14869).

ELISAs. ELISAs were performed as previously described (30). Briefly, wells of 96-well microtiter plates (Maxisorp for AM, Immulon $2 \mathrm{HB}$ for proteins, Fisher Scientific) were coated with AM at $10 \mu \mathrm{g} /$ $\mathrm{mL}$ or Mtb proteins at $4 \mu \mathrm{g} / \mathrm{mL}(50 \mu \mathrm{L} /$ well). Serum samples (1:50 or 1:100 dilution) were added in duplicates to the antigen-coated wells, and the bound Abs detected with protein G-alkaline phosphatase (AP) (1:2500; EMD Millipore) or goat anti-human IgG-AP for IgG detection (1:4000; SouthernBiotech), followed by $p$-nitrophenyl phosphate substrate. Secondary Abs for IgG subclass detection were 1:1000 diluted mouse anti-human IgG1-AP (clone 4E3), IgG2-AP (clone HP6002), IgG3-AP (clone HP6050), and IgG4-AP (clone HP6025; SouthernBiotech). The optical densities (ODs) were measured at $405 \mathrm{~nm}$. Negative controls were processed in duplicates as described above, except for the addition of serum. The murine anti-AM mAb CS35 (BEI Resources; NR-13811), anti-GroEL2 mAb IT13 (BEI Resources; NR-13825), and rabbit polyclonal anti-CFP10 (BEI Resources; NR-13801) were used as positive controls and detected with AP-conjugated goat anti-mouse IgG or goat anti-rabbit IgG (SouthernBiotech), respectively.

AM microarrays. A panel of 29 AM fragments (corresponding to motifs at the nonreducing terminus of the molecule) that have previously been shown to be recognized by anti-AM/LAM Abs and 1 fragment of $\alpha$-glucan (as control) were synthesized, and coupled to BSA via a squarate linker $(27,28,39)$. AM microarrays were processed as previously described (16). Briefly, after blocking with 3\% BSA/PBS, the arrays were incubated with diluted sera, a low-anti-AM-titer serum from a TST, non-BCG-vaccinated US-born volunteer (negative control), the purified anti-AM IgG $(5 \mu \mathrm{g} / \mathrm{mL})$, and the murine mAb CS35 $(5 \mu \mathrm{g} / \mathrm{mL}$; positive control). Abs were detected by goat anti-human biotin-labeled IgG (Jackson ImmunoResearch Laboratories Inc.) or goat anti-mouse biotin-labeled IgG (SouthernBiotech), followed by a streptavidin probe tagged with SureLightP3 Cy5 (Cayman Chemical). The slides were scanned using the GenePix 4000 Microarray scanner system (Molecular Devices), and images analyzed by the image-processing software The Institute for Genomics Research Spotfinder $(16,60)$. The MFI, representing AM epitope-specific Ab responses, was the measured median pixel intensity minus the background pixel intensity. Glycan array data were deposited in the NCBI Gene Expression Omnibus (GEO) database with accession number GSE141679.

Phagocytosis assay. A phagocytosis assay was performed essentially as previously described (16). Briefly, human THP-1 monocytic leukemia cells (ATCC) were cultured as recommended, differentiated into macrophages by phorbol 12-myristate 13-acetate, infected with FITC-conjugated Mtb H37Ra at an MOI of 20, and coincubated with $10 \%$ heat-inactivated sera for 3 hours. Note that we coincubated macrophages, sera, and Mtb to allow for the processing of multiple samples under the same conditions and have previously shown that this approach is comparable to preopsonizing mycobacteria before infection (16). After washing of the cells and quenching of the fluorescence from the noninternalized membrane-bound FITC-labeled H37Ra with trypan blue, the Mtb phagocytosis was evaluated by flow cytometry. To validate our results, we further investigated phagocytosis rates in human monocyte-derived macrophages (MDMs) from a healthy BCG-vaccinated but TST volunteer. Plated into 48 wells at $1 \times 10^{6}$ per well, PBMCs were differentiated into MDMs by being cultured in 10 $\mathrm{ng} / \mathrm{mL}$ macrophage colony-stimulating factor for 7 days, followed by infections with Mtb at MOI 10. For Fc $\gamma$ R blocking experiments, $10 \mu \mathrm{g} /$ $\mathrm{mL}$ mouse anti-human CD64 (clone 10.1, BD Pharmingen), mouse anti-human CD32 (clone AT10, AbD Serotec), mouse anti-human CD16 (clone 3G8, BD Pharmingen), or a control mouse mAb IgG1 (clone MOPC-21, Thermo Fisher Scientific) was added to the cells before infection for 1 hour and during coincubation with heat-inactivated sera.

Mtb intracellular growth inhibition assay. THP-1 cells were plated and differentiated into macrophages (16). The cells were infected with Mtb H37Rv (MOI 1) and coincubated with 10\% heat-inactivated sera for 2 hours, followed by washing. To calculate the Mtb intracellular growth in relation to amount phagocytosed, cells were lysed first after 2 hours on day 1, and then on day 3. Bacteria were numerated by plating of serial dilutions in duplicates on agar plates. Colony-forming units (CFU) were counted after incubation at $37^{\circ} \mathrm{C}$ for $4-6$ weeks, and the average $\mathrm{CFU}$ from triplicate wells calculated.

Assessment of phagosome-lysosomal fusion. Differentiated THP-1 cells were infected with Alexa Fluor 488-conjugated H37Rv at an MOI of 5 and coincubated with $10 \%$ heat-inactivated serum for 3 hours. To assess the amount of phagosome-lysosomal fusion, the cells were washed and probed with 100 nM LysoTracker Red DND-99 (Life Technologies) for 1 hour (16). A range of $150-400 \mathrm{H} 37 \mathrm{Rv}$ phagosomes, on images taken by a Leica SP8 confocal microscope, were counted to quantify the percentage of phagosomes colocalizing with the LysoTracker. 
Conjugation of AM to streptavidin Dynabeads. To be able to conjugate AM to magnetic Dynabeads, it was first activated by 1-cyano-4-dimethylaminopyridinium tetrafluoroborate (CDAP) and then conjugated to biotin (61). Briefly, AM dissolved in $\mathrm{H}_{2} \mathrm{O}$ at $1 \mathrm{mg} / \mathrm{mL}$ was added to CDAP (freshly prepared in Acetonitrile) to a final ratio of 1 $\mathrm{mg}$ of AM per $0.36 \mathrm{mg}$ of CDAP, followed by biotinylation to a final ratio of $1 \mathrm{mg}$ of AM per $0.57 \mathrm{mg}$ of EZ-Link Amine-PEG3-Biotin (Thermo Fisher Scientific). After termination of the reaction by addition of $25 \mathrm{mM}$ of glycine, the excess biotin was removed by dialysis against PBS, and the biotin-conjugated AM coupled to magnetic Dynabeads M-270 Streptavidin (Thermo Fisher Scientific) according to the manufacturer's instructions.

Selective serum depletion of Abs against AM or the Mtb surface. Diluted sera (1:5 in RPMI medium for heat-inactivated sera or in PBS for non-heat-inactivated sera, depending on downstream applications) were incubated with AM-coupled or control (BSA-coupled) Dynabeads at $4^{\circ} \mathrm{C}$ overnight, followed by magnetic separation from Dynabeads. Both anti-AM Ab-depleted and nondepleted sera were passed through a $0.22-\mu \mathrm{m}$ Spin-X filter (Corning Costar) for sterilization. To deplete Abs against the Mtb surface, H37Rv bacteria grown without detergent were pelleted and washed, incubated with the diluted sera at $37^{\circ} \mathrm{C}$ for 45 minutes, and pelleted down. The supernatants, representing the sera depleted by Abs against the Mtb surface, were then collected. This depletion process was performed twice or, if needed, 3 times before the depleted sera were passed through a $0.22-\mu \mathrm{m}$ Spin-X filter and taken out of the BSL-3 laboratory.

Purification of total IgG and anti-AM IgG. Total IgG, isolated from serum or plasma via protein $G$ agarose (Thermo Fisher Scientific), was incubated with CDAP-activated AM (61) conjugated to CarboxyLink Coupling Resins (Thermo Fisher Scientific). To generate AM-conjugated resin, the CDAP-activated AM was added into $0.5 \mathrm{~mL}$ resin in 0.15 M HEPES buffer and incubated at $4^{\circ} \mathrm{C}$ overnight on a rotator. The reaction was quenched with 0.5 $\mathrm{M}$ ethanolamine for 1 hour. Afterward, the AM-conjugated resin was packed into a 2-mL disposal column and washed with PBS. The flow-through was collected, and the column was sequentially washed with PBS/0.05\% Tween-20 and PBS. The anti-AM IgG was eluted in elution buffer (Thermo Fisher Scientific) and neutralized with $1 \mathrm{M}$ Tris, $\mathrm{pH}$ 8. Isolated anti-AM IgG was bufferexchanged into PBS or NS, and the concentration was quantified by an in-house human IgG ELISA.

Immunofluorescence. Mtb $\mathrm{H} 37 \mathrm{Rv}$ bacteria, grown without detergent, were fixed with $2 \%$ formaldehyde, placed on poly-Llysine slides (MilliporeSigma), and blocked with 1\% BSA/PBS (16). Human sera, nondepleted or anti-AM Ab depleted (1:100), or 5 $\mu \mathrm{g} / \mathrm{mL}$ purified anti-AM IgG diluted in blocking buffer were added and slides incubated at $4^{\circ} \mathrm{C}$ overnight. After washing, TRITClabeled goat anti-human IgG $(4 \mu \mathrm{g} / \mathrm{mL}$; SouthernBiotech) was added for 1.5 hours at room temperature in the dark. After washing, the slides were mounted with $90 \%$ glycerol, and viewed with a Zeiss observer microscope.

Murine infection. C57BL/6 female mice (7-8 weeks old; Charles River) were injected i.p. with $8 \mu \mathrm{g}$ anti-AM IgG in $100 \mu \mathrm{L}$ NS 1 day before and $4 \mu \mathrm{g} 1$ day after Mtb infection. The dose was based on approximately $0.3 \%$ of human IgG in our high-titer subjects being anti-AM IgG, and the human anti-AM IgG was administered twice to result in a comparable serum level in the mice after tissue distribution. Control mice received an irrelevant human mAb IgG1 (to a flavivirus) or NS at the same dose and/or volume. For the passive transfer experiment with anti-AM IgG from a TB patient, an additional injection of $4 \mu \mathrm{g}$ Ab i.p. was given 1 week after infection. Using a whole-body exposure aerosol chamber (Mechanical Engineering Workshop) custom-fitted to a class III biosafety cabinet (Baker), mice were infected aerogenically with approximately 10-20 CFU of Mtb (Erdman). During necropsy, the lungs were aseptically removed, homogenized in PBS, serially diluted, and plated on Middlebrook $7 \mathrm{H} 10$ agar. The CFUs were counted after 4 and 6 weeks of incubation at $37^{\circ} \mathrm{C}$.

Statistics. Statistical analysis was performed using Prism software version 7 (GraphPad) and Stata software version 15/IC (StataCorp). For ordinal data, we used Pearson $\chi^{2}$ or, when indicated, the Fisher exact test. Depending on data distribution, we used nonparametric tests (Kruskal-Wallis for unpaired and Friedman test for paired overall group comparison, Mann-Whitney $U$ test for 2-group comparison, and Wilcoxon matched-pairs signed-rank test for data comparison within the same group) or parametric tests (1-way ANOVA for overall group differences and $t$ tests for 2-group comparison, with Welch's correction if variances were unequal). Given the very limited number of a priori hypotheses being tested and the exploratory nature of this study, our main goal was to present the data, along with summaries and $P$ values for prespecified contrasts of interest, with no adjustment for multiple comparisons. Associations were assessed by Spearman's rank correlation. A 2-tailed $\alpha$ less than 0.05 was considered statistically significant.

Study approval. The study was approved by the institutional review board of the Albert Einstein College of Medicine. All human subjects gave written informed consent before enrollment. The animal studies were approved by the Institutional Animal Care and Use Committee at the Albert Einstein College of Medicine.

\section{Author contributions}

TC, CB, and JMA developed the research question and designed studies and experiments; TC, CB, YL, SS, and JX conducted experiments; TC, CB, and YL acquired data; TC, CB, EI, TLL, JC, and JMA analyzed data; MJ, TLL, ERJA, and JC provided reagents or human samples; and TC and JMA wrote the manuscript. All authors reviewed, edited, discussed, and approved the final version of the manuscript.

\section{Acknowledgments}

This work was supported in part by funds from the NIH (AI146329, AI127173, and AI117927 to JMA, AI139297 to JC, and 1ULTR002556 and P30CA013330 to the Albert Einstein College of Medicine). The work was further supported by funding from the Aeras TB Vaccine Foundation, the Bill and Melinda Gates Foundation, the Alberta Glycomics Centre, the Canadian Institutes for Health Research, and the Canadian Glycomics Network.

Address correspondence to: Jacqueline M. Achkar, Albert Einstein College of Medicine, 1300 Morris Park Avenue, Block Building, Room 115, Bronx, New York 10461, USA. Phone: 718.430.8763; Email: jacqueline.achkar@einsteinmed.org. 
1. World Health Organization (WHO). Tuberculosis vaccine development. http://www.who.int/ immunization/research/development/tuberculosis/en/. Updated January 16, 2020. Accessed January $27,2020$.

2. WHO. Executive summary. In: WHO, ed., Global Tuberculosis Report 2018. Geneva, Switzerland: World Health Organization; 2018:1-2.

3. Houben RM, Dodd PJ. The global burden of latent tuberculosis infection: a re-estimation using mathematical modelling. PLoS Med. 2016;13(10):e1002152.

4. Izzo AA. Tuberculosis vaccines - perspectives from the NIH/NIAID Mycobacteria vaccine testing program. Curr Opin Immunol. 2017;47:78-84.

5. Simmons JD, et al. Immunological mechanisms of human resistance to persistent Mycobacterium tuberculosis infection. Nat Rev Immunol. 2018;18(9):575-589.

6. Achkar JM, Prados-Rosales R. Updates on antibody functions in Mycobacterium tuberculosis infection and their relevance for developing a vaccine against tuberculosis. Curr Opin Immunol. 2018;53:30-37.

7. Sia JK, Georgieva M, Rengarajan J. Innate immune defenses in human tuberculosis: an overview of the interactions between Mycobacterium tuberculosis and innate immune cells. J Immunol Res. 2015;2015:747543.

8. Li H, Javid B. Antibodies and tuberculosis: finally coming of age? Nat Rev Immunol. 2018;18(9):591-596.

9. Van Der Meeren O, et al. Phase $2 \mathrm{~b}$ controlled trial of $\mathrm{M} 72 / \mathrm{ASO1}_{\mathrm{E}}$ vaccine to prevent tuberculosis. $N$ Engl JMed. 2018;379(17):1621-1634.

10. Nemes E, et al. Prevention of M. tuberculosis infection with $\mathrm{H} 4$ :IC31 vaccine or BCG revaccination. N Engl JMed. 2018;379(2):138-149.

11. Prados-Rosales R, et al. Enhanced control of Mycobacterium tuberculosis extrapulmonary dissemination in mice by an arabinomannan-protein conjugate vaccine. PLoS Pathog. 2017;13(3):e1006250.

12. Casadevall A. Antibody-based vaccine strategies against intracellular pathogens. Curr Opin Immunol. 2018;53:74-80.

13. Lu LL, Suscovich TJ, Fortune SM, Alter G. Beyond binding: antibody effector functions in infectious diseases. Nat Rev Immunol. 2018;18(1):46-61.

14. Bournazos S, Wang TT, Ravetch JV. The role and function of Fcy receptors on myeloid cells. Microbiol Spectr. 2016;4(6):10.1128/microbiolspec. MCHD-0045-2016.

15. de Vallière S, Abate G, Blazevic A, Heuertz RM, Hoft DF. Enhancement of innate and cell-mediated immunity by antimycobacterial antibodies. Infect Immun. 2005;73(10):6711-6720.

16. Chen T, et al. Association of human antibodies to arabinomannan with enhanced mycobacterial opsonophagocytosis and intracellular growth reduction. J Infect Dis. 2016;214(2):300-310.

17. Lu LL, et al. A functional role for antibodies in tuberculosis. Cell. 2016;167(2):433-443.e14.

18. Zimmermann N, et al. Human isotype-dependent inhibitory antibody responses against Mycobacterium tuberculosis. EMBO Mol Med. 2016;8(11):1325-1339.

19. Li H, et al. Latently and uninfected healthcare workers exposed to TB make protective antibodies against Mycobacterium tuberculosis. Proc Natl Acad Sci U S A. 2017;114(19):5023-5028.

20. Astronomo RD, Burton DR. Carbohydrate vaccines: developing sweet solutions to sticky situations? Nat Rev Drug Discov. 2010;9(4):308-324.

21. Colombo C, Pitirollo O, Lay L. Recent advances in the synthesis of glycoconjugates for vaccine development. Molecules. 2018;23(7):E1712.

22. Anish C, Schumann B, Pereira CL, Seeberger $\mathrm{PH}$. Chemical biology approaches to designing defined carbohydrate vaccines. Chem Biol. 2014;21(1):38-50.

23. Daffé M, Etienne G. The capsule of Mycobacterium tuberculosis and its implications for pathogenicity. Tuber Lung Dis. 1999;79(3):153-169.

24. Stokes RW, Norris-Jones R, Brooks DE, Beveridge TJ, Doxsee D, Thorson LM. The glycan-rich outer layer of the cell wall of Mycobacterium tuberculosis acts as an antiphagocytic capsule limiting the association of the bacterium with macrophages. Infect Immun. 2004;72(10):5676-5686.

25. Ortalo-Magné A, Dupont MA, Lemassu A, Andersen AB, Gounon P, Daffé M. Molecular composition of the outermost capsular material of the tubercle bacillus. Microbiology (Reading, Engl).1995;141(pt 7):1609-1620.

26. Angala SK, Belardinelli JM, Huc-Claustre E, Wheat WH, Jackson M. The cell envelope glycoconjugates of Mycobacterium tuberculosis. Crit Rev Biochem Mol Biol. 2014;49(5):361-399.

27. Lak P, Makeneni S, Woods RJ, Lowary TL. Specificity of furanoside-protein recognition through antibody engineering and molecular modeling. Chemistry. 2015;21(3):1138-1148.

28. Murase T, et al. Structural insights into antibody recognition of mycobacterial polysaccharides. JMol Biol. 2009;392(2):381-392.

29. Choudhary A, et al. Characterization of the antigenic heterogeneity of lipoarabinomannan, the major surface glycolipid of Mycobacterium tuberculosis, and complexity of antibody specificities toward this antigen. J Immunol. 2018;200(9):3053-3066.

30. Yu X, Prados-Rosales R, Jenny-Avital ER, Sosa K, Casadevall A, Achkar JM. Comparative evaluation of profiles of antibodies to mycobacterial capsular polysaccharides in tuberculosis patients and controls stratified by HIV status. Clin Vaccine Immunol. 2012;19(2):198-208.

31. Teitelbaum $\mathrm{R}$, et al. A mAb recognizing a surface antigen of Mycobacterium tuberculosis enhances host survival. Proc Natl Acad Sci U S A. 1998;95(26):15688-15693.

32. Hamasur B, Haile M, Pawlowski A, Schroder U, Kallenius G, Svenson SB. A mycobacterial lipoarabinomannan specific monoclonal antibody and its $\mathrm{F}(\mathrm{ab}$ ') fragment prolong survival of mice infected with Mycobacterium tuberculosis. Clin Exp Immunol. 2004;138(1):30-38.

33. Glatman-Freedman A, et al. Antigenic evidence of prevalence and diversity of Mycobacterium tuberculosis arabinomannan. JClin Microbiol. 2004;42(7):3225-3231.

34. Hamasur B, et al. Mycobacterium tuberculosis arabinomannan-protein conjugates protect against tuberculosis. Vaccine. 2003;21(25-26):4081-4093.

35. Brown RM, et al. Lipoarabinomannan-reactive human secretory immunoglobulin A responses induced by mucosal bacille Calmette-Guérin vaccination. JInfect Dis. 2003;187(3):513-517.

36. Lewinsohn DM, et al. Official American Thoracic Society/Infectious Diseases Society of America/ Centers for Disease Control and Prevention Clinical Practice Guidelines: Diagnosis of Tuberculosis in Adults and Children. Clin Infect Dis. 2017;64(2):111-115.

37. Guirado E, Schlesinger LS, Kaplan G. Macrophages in tuberculosis: friend or foe. Semin Immunopathol. 2013;35(5):563-583.

38. Berrington WR, Hawn TR. Mycobacterium tuberculosis, macrophages, and the innate immune response: does common variation matter? Immunol Rev. 2007;219:167-186.

39. Zheng RB, et al. Insights into interactions of mycobacteria with the host innate immune system from a novel array of synthetic mycobacterial glycans. ACS Chem Biol. 2017;12(12):2990-3002.

40. North RJ, Izzo AA. Mycobacterial virulence. Virulent strains of Mycobacteria tuberculosis have faster in vivo doubling times and are better equipped to resist growth-inhibiting functions of macrophages in the presence and absence of specific immunity. JExp Med. 1993;177(6):1723-1733.

41. Mukherjee J, Zuckier LS, Scharff MD, Casadevall A. Therapeutic efficacy of monoclonal antibodies to Cryptococcus neoformans glucuronoxylomannan alone and in combination with amphotericin B. Antimicrob Agents Chemother. 1994;38(3):580-587.

42. Charles River Laboratories International Inc. Proteus mirabilis - Technical Sheet. https:// www.criver.com/sites/default/files/resources/ ProteusmirabilisTechnicalSheet.pdf. Accessed January 27, 2020.

43. Moxon ER, Kroll JS. The role of bacterial polysaccharide capsules as virulence factors. Curr Top Microbiol Immunol. 1990;150:65-85.

44. Kang PB, et al. The human macrophage mannose receptor directs Mycobacterium tuberculosis lipoarabinomannan-mediated phagosome biogenesis. J Exp Med. 2005;202(7):987-999.

45. Joller N, et al. Antibodies protect against intracellular bacteria by Fc receptor-mediated lysosomal targeting. Proc Natl Acad Sci U S A. 2010;107(47):20441-20446.

46. O'Shea MK, et al. Immunological correlates of mycobacterial growth inhibition describe a spectrum of tuberculosis infection. Sci Rep. 2018;8(1):14480.

47. Cho YS, et al. Deciphering the proteome of the in vivo diagnostic reagent "purified protein derivative" from Mycobacterium tuberculosis. Proteomics. 2012;12(7):979-991.

48. Vidarsson G, Dekkers G, Rispens T. IgG subclasses and allotypes: from structure to effector functions. Front Immunol. 2014;5:520.

49. Costello AM, et al. Does antibody to mycobacterial antigens, including lipoarabinomannan, limit dissemination in childhood tuberculosis? Trans $R$ Soc Trop Med Hyg. 1992;86(6):686-692.

50. Logan E, et al. Elevated IgG responses in infants are associated with reduced prevalence of $\mathrm{Myco}$ bacterium tuberculosis infection. Front Immunol. 2018;9:1529.

51. Aase A, Michaelsen TE. Opsonophagocytic activity induced by chimeric antibodies of the 
four human IgG subclasses with or without help from complement. Scand J Immunol. 1994;39(6):581-587.

52. Shackelford PG, Polmar SH, Mayus JL, Johnson WL, Corry JM, Nahm MH. Spectrum of IgG2 subclass deficiency in children with recurrent infections: prospective study. JPediatr. 1986;108(5 pt 1):647-653.

53. Kuijpers TW, Weening RS, Out TA. IgG subclass deficiencies and recurrent pyogenic infections, unresponsiveness against bacterial polysaccharide antigens. Allergol Immunopathol (Madr). 1992;20(1):28-34.

54. Beenhouwer DO, Yoo EM, Lai CW, Rocha MA, Morrison SL. Human immunoglobulin G2 (IgG2) and IgG4, but not IgG1 or IgG3, protect mice against Cryptococcus neoformans infection. Infect Immun . 2007;75(3):1424-1435.

55. Ackerman ME, et al. A robust, high-throughput assay to determine the phagocytic activity of clinical antibody samples. JImmunol Methods. 2011;366(1-2):8-19.

56. Fleit HB, Kobasiuk CD. The human monocyte-like cell line THP-1 expresses Fc gamma RI and Fc gamma RII. J Leukoc Biol. 1991;49(6):556-565.

57. Srivastava S, Ernst JD, Desvignes L. Beyond macrophages: the diversity of mononuclear cells in tuberculosis. Immunol Rev. 2014;262(1):179-192.

58. Joosten SA, et al. Patients with tuberculosis have a dysfunctional circulating B-cell compartment, which normalizes following successful treatment. PLoS Pathog. 2016;12(6):e1005687.

59. Paul S, Laochumroonvorapong P, Kaplan G. Comparable growth of virulent and avirulent Mycobacterium tuberculosis in human macrophages in vitro. J Infect Dis. 1996;174(1):105-112.

60. Saeed AI, et al. TM4: a free, open-source system for microarray data management and analysis. BioTechniques. 2003;34(2):374-378.

61. Lees A, Nelson BL, Mond JJ. Activation of soluble polysaccharides with 1-cyano-4-dimethylaminopyridinium tetrafluoroborate for use in protein-polysaccharide conjugate vaccines and immunological reagents. Vaccine. 1996;14(3):190-198. 